\title{
Gewerkschaften in der Volksrepublik China
}

\author{
Von Liu Jen-Kai
}

Dieser Artikel ist als Einführung in die Geschichte der Gewerkschaften in der VR China konzipiert; er kann deshalb keine detaillierte Untersuchung bestimmter Einzelprobleme geben, sondern nur einen allgemeinen Uberblick über den historischen Verlauf der Gewerkschaftsbewegung nach der Befreiung 1949 und die allgemeine Problematik der Gewerkschaft in der sozialistischen chinesischen Gesellschaft vermitteln.

\section{Vorgeschichte ${ }^{1}$}

Vor der Gründung von modernen Gewerkschaften in China hatten verschiedenartige - zumeist traditionale - Organisationen die Interessen der Arbeiter wahrgenommen, wie die Gilden, Landsmannschaften und Geheimgesellschaften. Unter den letzteren ist die ,,Gesellschaft des kleinen Messers" in Shanghai hervorzuheben, die die Seeleute vertrat, ferner die „,Grüne Bande", die sich um 200000 Transportarbeiter kümmerte, sowie die für den Kampf gegen die Fremdherrschaft der Mandschus (diese letzte Dynastie in China stürzte 1911 und machte der Republik Platz) eintretende „Rote Bande“. 1914 findet man bereits unter den Seeleuten in Hongkong und Shanghai gemischte Arbeitnehmer- und Arbeitgeberorganisationen. Reine Vertretungen von Arbeitern kann man sogar schon 1906 ausmachen. Die Tätigkeit all dieser meist illegalen Organisationen läßt sich daran ablesen, daß zwischen 1895 und 1918 etwa 200 Streiks stattfanden².

Der Kommunistischen Partei Chinas (KPCh) gebührt das Verdienst der Gründung moderner Gewerkschaftsorganisationen. Erfahrungen dazu hatte man im Ausland gesammelt, wo es etwa 3000 ,,Arbeits- und Studium“-Studenten gab. Zu ihnen gehörten u. a. spätere Gewerkschaftsführer wie Li Lisan, Liu Shaoqi, Cai Hesen. Eine weitere Hilfe kam von seiten der Komintern. Im Anschluß an die ,,4.-Mai-Bewegung“ von 1919 war es im republikanischen China zum Aufbau von ,A Arbeiterschulen“ durch marxistische Studenten gekommen. Im Herbst 1921 wurde dann nach dem 1. Parteitag der KPCh in Shanghai das Sekretariat der chinesischen Arbeitervereinigungen gebildet; die Zeitschrift dieser KP-Organisation hieß „Arbeiterwochenschrift" (laodong zhoukan). Die Versuche der Organisierung der Arbeiter waren so erfolgreich, daß schon am 1. 5. 1922 in Kanton der 1. Nationale Arbeiterkongreß abgehalten werden konnte. 162 Delegierte vertraten 70000 organisierte Arbeiter von über 100 Gewerkschaften $^{3}$. Hauptpunkte waren der Beschluß über die Prinzipien gewerkschaftlicher Organisation und die Forderungen nach dem Acht-Stunden-Tag sowie einer Arbeitsgesetzgebung. Das Sekretariat der chinesischen Arbeitervereinigungen wurde beauftragt, bis zur Gründung eines nationalen Gewerkschaftsverbandes als zentrales Verbindungsbüro aller Gewerkschaften zu fungieren und die Einberufung des 2. Arbeiterkongresses vorzubereiten. Die Zeit bis 1923, als die Kommunisten in der Arbeiterbewegung auf Untergrundarbeit umschalten mußten, sah große Streiks, so den Streik der Hongkonger Seeleute von Januar bis März 1922 und den der Eisenbahner an der Peking-Hankou-Eisenbahn vom 7. 2. 1923. Der 2. Nationale Arbeiterkongreß fand a m 1. 5.1925 in Kanton statt. 280 Delegierte repräsentierten nominell 540000 Mitglieder, die in 166 Gewerkschaften organisiert waren ${ }^{4}$.

Ein Abkürzungsverzeichnis befindet sich auf Seite 330.

1 Zur Vorgeschichte s. J. Chesneaux, The Chinese Labor Movement, 1917-1927, Stanford 1968; Nym Wales, The Chinese Labor Movement, New York 1945; P. Schier, , Die chinesische Arbeiterbewegung“, in: R. Lorenz (Hrsg.), Zur Sozialgeschichte der chinesischen Revolution (1911-1949) (Frankfurt/Main 1977), S. 257-350; Deng Zhongxia, Anfänge der chinesischen Arbeiterbewegung 1919-1926, Hamburg 1975.

2 s. Schier, a. a. O., S. 276

3 s. ebenda, S. 297.

4 s. ebenda, S. 309. 
Dieser Kongreß brachte die Gründung des Allchinesischen Gewerkschaftsbundes (ACGB); an der Spitze des Exekutivkomitees stand ein Seemann, Lin Weimin, einer der beiden Stellvertreter wurde der spätere Staatspräsident der VR China, Liu Shaoqi. Kontakte zur internationalen kommunistischen Gewerkschaftsbewegung wurden jetzt hergestellt. Das Jahr 1925 erlebte einen Aufschwung in der Gewerkschaftsarbeit und demzufolge eine Welle von Streiks. Der ,30.-Mai-Zwischenfall“ in Shanghai führte zu Kämpfen gegen britische und japanische Imperialisten, der über ein Jahr dauernde Hongkong-Kanton-Streik (vom Juni 1925 bis zum Oktober 1926) erfaßte bis 250000 Arbeiter. Am 1. 5. 1926 fand der 3. Nationale Gewerkschaftskongreß ebenfalls in Kanton statt. 502 Delegierte vertraten nun 1240000 Mitglieder in 699 Gewerkschaften ${ }^{5}$. Die Arbeiter unterstützten Tschiang Kaischek's Nordfeldzug gegen die Militärmachthaber u. a. durch eine Fülle von Streiks. Sichtbarster Ausdruck der Stärke der Gewerkschaften war der Aufstand von Shanghai, bei dem am 21. 3. 1927 die Arbeiter die Stadt besetzten und den Einmarsch der Guomindang-Truppen vorbereiteten. Tschiang Kai-schek's Dank dafür bestand in der brutalen Zerschlagung der Shanghaier Arbeiterbewegung und der Gewerkschaftsorganisationen in seinem Machtbereich am 12. April und danach. Kurz zuvor, im März 1927, wurde auch der 4. Nationale Gewerkschaftskongreß in Wuhan abgehalten, der auf eine Mitgliederzahl von 2900000 sehen konnte ${ }^{6}$. Der 5. Nationale Gewerkschaftskongreß fand im geheimen im September 1929 in Shanghai statt. Tschiang Kai-schek oktroyierte den Arbeitern ,,Gelbe Gewerkschaften“ auf. Eine die wahren Interessen der Arbeiter vertretende Gewerkschaftsarbeit der Kommunisten in den Städten war aufgrund des Drucks der Guomindang und dann der japanischen Imperialisten immer schwieriger geworden. Nicht ganz so viele Probleme hatten die Gewerkschaften in den Sowjetgebieten. 1934 zählte man dort 300000 Mitglieder, in Yan'an war diese Zahl 1937 durch die Verluste während des Langen Marsches auf 13000 gesunken. 1943 sprach man dagegen schon wieder von 1000000 Mitgliedern in den von der KPCh kontrollierten Gebieten ${ }^{7}$. Nach langer Pause trat im August 1948 in der Stadt Harbin in Nordost-China der 6. Nationale Gewerkschaftskongreß zusammen, auf dem 518 Delegierte 2830000 Mitglieder repräsentierten ${ }^{8}$. Vorsitzender wurde Chen Yun, de facto hatte jedoch der neben Liu Ningyi und Zhu Xuefan stellvertretende Vorsitzende Li Lisan die Leitung inne. Li, gegen dessen als ,,Putschismus“ verurteilte Politik der Eroberung großer Städte im Jahr 1930 sich der 3. Linienkampf in der Geschichte der KPCh wandte, war ein bekannter Gewerkschaftsführer, der nach 15jährigem Aufenthalt in der Sowjetunion nach China zurückgekehrt war.

\section{Die theoretische Grundlage kommunistischer Gewerkschaften}

In China gelten für alle Bereiche in Politik und Wirtschaft die Prinzipien des Marxismus-Leninismus. Die KPCh gilt demgemäß als Avantgarde der Arbeiter. ,,Die den Kern bildende Kraft, die unsere Sache führt, ist die Kommunistische Partei Chinas. Die theoretische Grundlage, von der sich unser Denken leiten läßt, ist der Marxismus-Leninismus. "9 In den Aussagen über die Funktion der Gewerkschaften berufen sich die Chinesen stets auf die

5 s. ebenda, S. 319

6 s. ebenda, S. 321

7 s.H. Martin/W. Bartke, Die Massenorganisationen in der Volksrepublik China, Mitteilungen des Instituts für Asienkunde Hamburg, Nr. 62 (Hamburg 1975), S. 72.

8 s. S. Peter, Zur Entwicklung der Gewerkschaften in der Volksrepublik China von 1949 bis zum 8. Allchinesischen Gewerkschaftskongreß 1957 (Magisterarbeit, Berlin 1974), S. 55.

9 s. Worte des Vorsitzenden Mao Tse-tung (Peking 1967), S. 1. 
marxistischen Klassiker. Die Partei als „,Vorhut der Arbeiterklasse“ und als ,,organisierte Abteilung der Arbeiterklasse "10 erstreckt ihre Führung auch auf die anderen Organisationen des Proletariats, von denen eine sehr wichtige die Gewerkschaft ist. Mao Zedong stellte fest: „,Die Partei ist die Avantgarde des Proletariats und die höchste Organisationsform des Proletariats, sie muß alle anderen Organisationen, wie Armee, Regierung und Massenorganisationen, führen. "11 Unter letztere fallen die Gewerkschaften, die von Lenin und Stalin als bedeutendes Instrument angesehen werden, mit dem die Partei Kontakt zu den Massen hält und ihre Politik an der Basis verwirklichen kann. Dabei ist die Gewerkschaftsorganisation formal parteilos, realiter aber eng mit der Partei verbunden. Lenin führte das in ,, Der , linke Radikalismus', die Kinderkrankheit im Kommunismus“ aus: ,,Die Partei stützt sich bei ihrer Arbeit unmittelbar auf die Gewerkschaften, die ... der Form nach parteilos sind. Faktisch bestehen alle leitenden Körperschaften der weitaus meisten Verbände und in erster Linie natürlich der Zentrale oder des Büros aller Gewerkschaften ganz Rußlands aus Kommunisten und führen alle Direktiven der Partei durch. Im großen und ganzen haben wir also einen der Form nach nicht kommunistischen, elastischen und verhältnismäßig umfassenden, überaus mächtigen proletarischen Apparat, durch den die Partei mit der Klasse und der Masse eng verbunden ist und durch den, unter der Führung der Partei, die Diktatur der Klasse verwirklicht wird. Ohne die engste Verbindung mit den Gewerkschaften, ohne ihre tatkräftige Unterstützung, ohne ihre selbstlose Arbeit beim Aufbau ..., hätten wir das Land selbstverständlich keine zweieinhalb Monate, geschweige denn zweieinhalb Jahre regieren und die Diktatur ausüben können. " 12 Stalin griff diese Überlegungen in , Ưber die Grundlagen des Leninismus“ auf. Die Partei bildet ,,als die beste Schule von Führern der Arbeiterklasse, dank ihrer Erfahrung und Autorität die einzige Organisation . . ., die fähig ist, die Leitung des Kampfes des Proletariats zu zentralisieren und auf diese Weise alle wie immer gearteten parteilosen Organisationen der Arbeiterklasse in Hilfsorgane und Transmissionsriemen zu verwandeln, die sie mit der Klasse verbinden . . . Das bedeutet nicht, daß die parteilosen Organisationen, die Gewerkschaften, Genossenschaften usw., der Leitung der Partei formal unterstellt sein müssen. Es handelt sich nur darum, daß die Parteimitglieder, die diesen Organisationen angehören, als zweifellos einflußreiche Menschen alle Mittel der Úberzeugung anwenden, damit die parteilosen Organisationen in ihrer Tätigkeit der Partei des Proletariats möglichst nahegebracht werden und freiwillig ihre politische Führung anerkennen. "13 Dagegen ,,ist die opportunistische Theorie von der ,Unabhängigkeit' und ,Neutralität' der parteilosen Organisationen, die unabhängige Parlamentarier und von der Partei losgelöste Journalisten, engstirnige Gewerkschaftler und verspießerte Genossenschaftler züchtet, völlig unvereinbar mit der Theorie und Praxis des Leninismus“"14. Mit der Ubernahme der politischen Macht durch das Proletariat entsteht auch ein Wandel in den Aktivitäten der Gewerkschaften, die nun die ,, wichtigsten Baumeister der neuen Gesellschaft" und „Baumeister des neuen Lebens" 15 werden. Sie sind ,,insbesondere nach der politischen Umwälzung . . . berufen, eine besonders große Rolle zu spielen, in der Politik die zentralste Stellung einzunehmen, in gewissem Sinn des Wortes zum politischen Hauptorgan zu werden“16. Die Arbeiter werden nun nicht mehr ,,zum Widerstand gegen die Staatsmacht“ mo-

\footnotetext{
10 J. Stalin, Uber die Grundlagen des Leninismus (Verlag für fremdsprachige Literatur, Moskau 1947), S. 87 und 89.

11 Zit. in: Ma Chungu, ,Gründlich die der Gewerkschaft gebührende Funktion entfalten“, ,,Rote Fahne“ (hongqi) 5/1978, S. 28.

12 Dietz Verlag Berlin 1968, S. 35 f.

13 Stalin, a. a. O., S. 93.

14 ebenda.

15 W. I. Lenin, Referat auf dem 2. Gesamtrussischen Gewerkschaftskongreß, Lenin Werke 28 (Dietz Verlag Berlin 1975), S. 438 und 440.

16 Lenin, ebenda, S. 430.
} 
bilisiert, sondern zur Unterstützung und Förderung der Maßnahmen ihrer eigenen Arbeitermacht, zur konsequenten Durchführung dieser Maßnahmen ${ }^{17}$. Die Aufgabe der Gewerkschaft besteht darin, die Werktätigen mit der Verwaltung, der Wirtschaftsführung und den Ideen des Kommunismus vertraut zu machen. „Einerseits sind die Gewerkschaften, die die Industriearbeiter in ihrer Gesamtheit erfassen und in die Reihen der Organisation einbeziehen, eine Organisation der machthabenden, herrschenden, regierenden Klasse, derjenigen Klasse, die den staatlichen Zwang ausübt. Aber das ist keine Organisation des Zwanges, das ist vielmehr eine erzieherische Organisation, eine Organisation der Heranziehung, der Schulung, das ist eine Schule, eine Schule der Verwaltung, eine Schule der Wirtschaftsführung, eine Schule des Kommunismus. “18 „Die Teilnahme der Gewerkschaften an der Wirtschaftsführung und die Heranziehung der breiten Massen zu dieser Arbeit durch sie ist zugleich das Hauptmittel des Kampfes gegen die Bürokratisierung des Wirtschaftsapparates . . und gibt die Möglichkeit, eine wirkliche Volkskontrolle über die Ergebnisse der Produktion zu schaffen."19 Die Gewerkschaften müssen ,,die Verbindung der Avantgarde", der Partei, ,,mit den Massen herstellen“ und ,,durch ihre tägliche Arbeit die Massen überzeugen“, denn ,,die Diktatur des Proletariats läßt sich nur verwirklichen durch eine Organisation, die das Proletariat in seiner Gesamtheit erfaßt " ${ }^{20}$ Es , ,ist eine Katastrophe unseres sozialistischen Aufbaus unvermeidlich, wenn der Transmissionsmechanismus zwischen der Kommunistischen Partei und den Massen - die Gewerkschaften - falsch aufgebaut ist oder nicht richtig funktioniert'‘21.

\section{Die Gewerkschaften bis zum 7. Nationalen Gewerkschaftskongreß 1953}

Nach dem Sieg der Revolution und der Errichtung der VR China am 1. 10. 1949 stellten sich dem jungen Staat in den ersten Jahren schon eine Fülle von Aufgaben, selbst gewählte und aufgezwungene, wie die Durchführung der Bodenreform, die Unterdrückung der Konterrevolution, der Widerstand gegen Amerika und die Hilfe für Korea, die „,Drei-Anti- und Fünf-Anti-Bewegung “. Zunächst war die Staatsform noch nicht wie später die Diktatur des Proletariats, sondern es handelte sich um einen alle demokratischen Klassen und Nationalitäten des Landes umfassenden Staat mit der demokratischen Diktatur des Volkes, unter Führung der Arbeiterklasse und auf der Grundlage des Bündnisses der Arbeiter und Bauern. Die ersten Jahre der VR China waren in der Wirtschaft dadurch gekennzeichnet, daß neben staatlichen und gemischt staatlich-privaten Betrieben weiterhin private Betriebe existierten. In behutsamen Schritten wurden auch diese Betriebe über dieForm gemischt staatlich-privater Betriebe schließlich in Gemeineigentum überführt. Die verschiedenen Arten des Eigentums an Produktionsmitteln machten natürlich auch entsprechend unterschiedliche Gewerkschaftsaktivitäten nötig.

Am 29. 6. 1950 wurde von der Zentralen Volksregierung das Gewerkschaftsgesetz ${ }^{22}$ erlassen, das in 26 Artikeln die allgemeinen Prinzipien (Art. 1-4), die Rechte und Pflichten (Art. 5-12), die Basisorganisationen (Art. 13-22) sowie den Fonds (Art. 23-25) bestimmte. Es legt fest, daß die Gewerkschaften Massenorganisationen der Arbeiterklasse auf freiwilliger Basis

\footnotetext{
17 Lenin, Úber die Heranziehung der Massen zur Leitung des Staates (Berlin 1965), S. 241; zit. in: Peter, a. a. O., S. 21.

18 Lenin, Uber die Gewerkschaftsbewegung, Bd. 1 (Berlin 1970), S. 304; zit. in: Peter, a. a. O., S. 22.

19 ebenda, S. 353; zit. in: Peter, a. a. O., S. 23.

20 Lenin, Uber die Heranziehung der Massen. ., a. a. O., S. 246; zit. in: Peter, a. a. O., S. 25.

21 Lenin, Uber die Rolle und die Aufgaben der Gewerkschaften unter den Verhältnissen der Neuen Okonomischen Politik, Lenin Werke 33 (Dietz Verlag Berlin 1977), S. 178.

22 Ubersetzung in: Th. H. E. Chen, The Chinese Communist Regime, Documents and Commentary (London 1967), S. 274-280.
} 
sind. Alle, bei denen der Lohn das einzige Mittel des Lebensunterhaltes darstellt, können sich gewerkschaftlich organisieren, ganz gleich, ob sie in Betrieben, Institutionen oder Schulen arbeiten. (Art. 1) Gewerkschaften sind gemäß dem Prinzip des demokratischen Zentralismus zu organisieren. (Art. 2) Höchstes Gremium ist der Allchinesische Gewerkschaftsbund. Die Artikel über Rechte und Pflichten der Gewerkschaften berücksichtigen staatliche sowie private Unternehmen. In den letzteren nahmen die Gewerkschaften an Konsultativkomitees von Arbeit und Kapital teil. Probleme, die in diesen Komitees nicht gelöst werden konnten, wurden gemäß den „,Bestimmungen zur Beilegung von Arbeitsstreitigkeiten“ an aus Partei- und Gewerkschaftsvertretern zusammengesetzte „,Arbeitsbüros“ verwiesen, so daß die letztgültige Entscheidung doch zugunsten der Arbeiter fiel. , Zur Sicherung der fundamentalen Interessen der Arbeiterklasse sollen die Gewerkschaften die folgenden Aktivitäten durchführen“: ,,1. Erziehung und Organisierung der Massen der Arbeiter und Angestellten, damit diese die Gesetze und Bestimmungen der Volksregierung unterstützen, die Politik der Volksregierung durchführen und die Staatsmacht des Volkes, die von der Arbeiterklasse geführt ist, konsolidieren; 2. Erziehung und Organisierung der Massen der Arbeiter und Angestellten, damit diese eine neue Einstellung gegenüber der Arbeit einnehmen, die Arbeitsdisziplin beachten sowie Arbeitswettbewerbskampagnen organisieren und andere Produktionskampagnen, um die Erfüllung der Produktionspläne zu gewährleisten; 3. Schutz des öffentlichen Eigentums, Widerstand gegen Korruption, Verschwendung und Bürokratie, Kampf gegen Saboteure in vom Staat oder von Kooperativen betriebenen Unternehmen sowie in Institutionen und Schulen; 4. Förderung der Politik der Entwicklung der Produktion und Zufriedenstellung von Arbeit und Kapital in privaten Unternehmen, Widerstand gegen Verletzungen der Gesetze und Erlasse der Regierung oder gegen die Produktion gerichtete Akte.“ (Art. 9) ,,Fabriken, Bergwerke, Firmen, Farmen, Institutionen' Schulen und andere produktive oder administrative Einheiten mit über 25 Arbeitern und Angestellten können ein Basisgewerkschaftskomitee errichten. " Bei unter 25 Personen wird ein Organisator gewählt, der die gleichen Rechte wie das Komitee hat. (Art. 13) Die Zahl der voll arbeitenden Gewerkschaftskader, die von der Produktion freigestellt sind, ist festgelegt; es soll einer auf 500 Arbeiter und Angestellte kommen mit einem Maximum von fünf solchen Kadern in Betrieben mit 2500 bis 4000 Arbeitern. Ist der Betrieb noch größer, kann ein Kader auf jeweils 2000 Personen hinzukommen. Der Fonds der Gewerkschaften setzt sich aus folgenden Quellen zusammen: 1. Mitgliedsbeitrag; 2. Leitung oder Besitzer muß der betreffenden Gewerkschaft eine 2 Prozent des Gesamtbetrages der Reallöhne entsprechende Summe zukommen lassen. 1,5 Prozent des Gesamtbetrages der Reallöhne werden zur Förderung kultureller und erzieherischer Aktivitäten bei den Arbeitern und Angestellten verwendet. 3. Einnahmen aus den von der Gewerkschaft geförderten kulturellen und sportlichen Aktivitäten; 4. Zuschüsse von den Volksregierungen der verschiedenen Ebenen. (Art. 24)

Die Gewerkschaftsstruktur sah in den frühen Jahren der VR China folgendermaßen aus: Bis 1950 existierten keine nationalen Gewerkschaften mit Ausnahme der Gewerkschaften der Seeleute und Eisenbahner. Am 7. 2. 1950 erfolgte in der ,Volkszeitung“ (rènmin ribao) ein Aufruf zur Gründung von zumindest zehn nationalen Industriegewerkschaften innerhalb von sechs Monaten. Ein solcher Gewerkschaftsapparat wurde erst mit Beginn des 1. Fünfjahresplans 1953 gefestigt. Anstelle von nationalen Einzelgewerkschaften gab es zunächst lokale, nach Arbeitsgebieten unterschiedene Gewerkschaften, die mit dem ACGB direkt affiliiert waren. Unterstellt waren sie den Gewerkschaftsräten auf Stadt-, Kreis- und Provinzebene. Oft waren die in diesen Räten arbeitenden Gewerkschaftler auch maßgebliche Leute in den betreffenden Parteikomitees. 
An Publikationen verfügten die Gewerkschaften über die „Arbeiterzeitung“" (gongren ribao) und die Monatszeitschrift „Chinesischer Arbeiter" (zhongguo gongren).

In den Monaten bis zum Sommer 1950 hatten die Gewerkschaften sich in den Betrieben aus Gründen des Aufbaus der zerrütteten Wirtschaft oft auf die Seite der Verwaltung geschlagen und gegen die Interessen der Arbeiter gerichtete Maßnahmen wie niedrigere Löhne und Wohlfahrtsleistungen gutgeheißen. Diese Haltung war sowohl in den privaten wie auch in den staatlichen Betrieben anzutreffen. Sie wurde als ,, Kommandierhaltung “ und ,,Bürokratismus" verurteilt. Nach dem Sommer verfielen die Gewerkschaften in das andere Extrem; statt Handlanger der Verwaltung zu sein, praktizierten sie nun Ớkonomismus, das heißt, sie unterstützten einseitig die materiellen Forderungen der Arbeiter, ohne die von der Partei vertretenen langfristigen Gesamtinteressen zu berücksichtigen. Grund für diesen Umschwung war die im August beginnende Ausrichtungsbewegung in den Gewerkschaften, nachdem Mao Zedong auf der 3. Plenartagung des Zentralkomitees der KPCh im Juni 1950 eine Ausrichtungsbewegung gefordert hatte.

Ein grundsätzliches Problem trat zutage. Li Lisan versuchte, für die Gewerkschaften eine gewisse Autonomie zu erreichen, weil er meinte, nur so auch unmittelbare Interessen der Arbeiter durchsetzen zu können. Dies führte zu Gegenmaßnahmen der Partei, die eine Polarisierung von unmittelbaren, kurzfristigen Interessen der Arbeiter und langfristigen Interessen des Staates befürchtete. In der Theorie ist es doch so, daß in einem Staat der demokratischen Diktatur des Volkes, der vom Proletariat mittels seiner Avantgarde, der Kommunistischen Partei, geführt wird und auch die Interessen der drei anderen Klassen (Bauern, Kleinbürgertum und nationale Bourgeoisie) vertritt, die Interessen der Arbeiter und des Staates identisch sein sollten. In der Praxis kommt es jedoch des öfteren zu Konflikten zwischen diesèn beiden Arten von Interessen. Es ist Aufgabe der Gewerkschaft, den Arbeitern verständlich zu machen, daß die langfristigen Interessen des Staates, der ja nicht nur die Interessen eines Teils der Gesellschaft, der Arbeiter, zu verfolgen hat, sondern das ganze Volk auf das Endziel einer kommunistischen Gesellschaft hin zu orientieren versucht, die übergreifenden Interessen sind, denen sich die kurzfristigen, individuellen Interessen von Arbeitern im Zweifelsfalle unterzuordnen haben. „Die unmittelbaren Teilinteressen der Arbeiterklasse müssen den langfristigen und umfassenden Interessen des Staates untergeordnet sein . . . Falls dagegen die langfristigen und umfassenden Interessen der Arbeiterklasse ihren unmittelbaren und Teilinteressen geopfert und Fehler des Ókonomismus begangen würden, würde die Gewerkschaft unvermeidlich eine enge, geteilte Berufsorganisation werden, und es wäre der Arbeiterklasse unmöglich, die glorreiche Zukunft des Kommunismus zu erreichen. " 23

In Reaktion auf die Autonomietendenz der Gewerkschaft hielten die Parteimitglieder im ACGB im Dezember 1951 eine erweiterte Sitzung ab über ,,die verschiedenen Meinungen hinsichtlich der Frage der Gewerkschaftsarbeit". Das Mitglied des Exekutivkomitees Li Fuchun kritisierte Li Lisan's Ökonomismus und Autonomiebestrebungen. Li wurde vorgeworfen, er sei dafür eingetreten, daß die Verwaltung die Interessen des Ganzen und die langfristigen Interessen sowie die Produktion vertreten solle, während sich die Gewerkschaften der individuellen und unmittelbaren Interessen sowie der Distribution annehmen sollten. ,,Einige führende Genossen im ACGB“, so hieß es, ,, hatten sogar die Führung der Partei über die Gewerkschaften geleugnet, . . . indem sie die Gewerkschaften als die höchste Organisa-

23 Lai Ruoyu auf dem 7. Nationalen Gewerkschaftskongreß 1953, zit. in: Peter S. H. Tang, Communist China Today, Vol. I, Domestic and Foreign Policies (Washington D. C. 1961), S. 377. 
tionsform der Arbeiterklasse ansahen. " ${ }^{24}$ Die Folge dieser massiven Kritik war, daß auf der 2. Plenartagung des Exekutivkomitees des ACGB im Februar 1953 Li Lisan abgewählt und etwa ein Drittel der Mitglieder dieses Exekutivkomitees ersetzt wurde.

Abgesehen von diesen Veränderungen an der Spitze war es schon 1952 zu einem erheblichen Wandel an den unteren Ebenen gekommen. 1952 hatte es nämlich die ,Fünf-Anti-Bewegung" gegeben, eine Massenkampagne, die sich in Industrie und Handel gegen Bestechung, Steuerhinterziehung, Diebstahl von Staatsvermögen, schlechte Arbeitsausführung wegen minderwertigen Materials und Wirtschaftsspionage wendete und die bürgerlichen Industriellen und Kaufleute im Visier hatte. Die Gewerkschaften waren maßgeblich an diesem Kampf beteiligt. In Shanghai zum Beispiel wurde einer von zehn Arbeitern in den dortigen privaten Firmen Aktivist, so daß er an den Arbeitsbrigaden teilnahm, die die Arbeiter während der ,,Fünf-Anti-Bewegung“" mobilisierten ${ }^{25}$. Viele neue Kader konnten aus den Reihen der Gewerkschaftsaktivisten gewonnen werden. So konnte die Nachrichtenagentur Neues China melden: ,Die neu gewählten Kader sind alle Gewerkschaftskader, Gewerkschaftsmitglieder oder Aktivisten, deren Klassenstandpunkt in der ,Fünf-Anti-Bewegung fest war, die positiv mit den Arbeitern verbunden waren und die standhaft gegen kriminelle Kapitalisten kämpften. “'26 1952 sollen 105240 Arbeiter Verwaltungsfunktionen übernommen haben, 7840 Arbeiter Direktoren oder Vizedirektoren geworden sein und fast 20000 Arbeiter eine Ausbildung zu technisch qualifizierten Kadern erhalten haben ${ }^{27}$.

Neben der ,Fünf-Anti-Bewegung“ hatten weitere Bewegungen in den Anfangsjahren der VR China eine gewaltige Erziehungsfunktion und trugen zur Erhöhung des Klassenbewußtseins der breiten Massen des Volkes bei, so die „,Bewegung zur Unterdrückung der Konterrevolution“'28, die ihren Höhepunkt im Frühjahr 1951 erlebte, und die in ihrem späteren Stadium erfolgende ,demokratische Reformbewegung“, die in Wirtschaftsunternehmen durchgeführt wurde. Die Absicht der Reformbewegung war es, ,,die Massen zu mobilisieren, damit sie die verbliebenen feudalen Kräfte innerhalb der Unternehmen zerschlagen, die Hindernisse in der Produktion zu beseitigen, das politische Bewußtsein der Massen der Arbeiter zu erhöhen, ihren Verantwortungssinn als Herren und ihre Produktionsbegeisterung zu erhöhen, wodurch günstige Bedingungen für die weitere Entwicklung der Produktion und das Starten der Produktionsbewegung im riesigen Ausmaß geschaffen werden“"29. Ferner sollten durch diese Bewegung, ,die alten Kräfte und alten Verwaltungssysteme, die die Arbeiter unterdrückt hatten“, vernichtet und „,ein neues Verwaltungssystem“ errichtet werden sowie „,Gewerkschaften, Fabrik-Leitungskomitees und Arbeiterkongresse“ ${ }^{\text {. }}$. Im Mai 1953 fand in Peking der 7. Nationale Gewerkschaftskongreß mit 800 Delegierten, die nun schon 10200000 Mitglieder von 180000 Gewerkschaften in 23 Nationalen Verbänden vertraten, statt ${ }^{31}$. Neuer Vorsitzender wurde Lai Ruoyu, der im Gegensatz zu seinem Vorgänger Li Lisan kein altgedienter Experte in der Gewerkschaftsarbeit war. Lai war schon 1952 Generalsekretär des ACGB geworden. Der Gewerkschaftskongreß wurde in einer Zeit einberufen, in der große Anstrengungen für die Industrialisierung des Landes und den

\footnotetext{
24 GRRB, 11. 2. 53; s. a. P. Harper, ,,The Party and the Unions in Communist China“, China Quarterly 37/1969, S. 95 f.

25 Zur ,Fünf-Anti-Bewegung“ in Shanghai s. J. Gardner, ,,The Wu-fan Campaign in Shanghai: A Study in the Consolidation of Urban Control", in: A. Doak Barnett (Hrsg.), Chinese Communist Politics in Action (Seattle 1969), S. 477-539.

26 Meldung vom 21. 5. 52.

27 s. A. Deter, Das neue China und die chinesischen Gewerkschaften (Berlin 1953), S. 50 f.; zit. in: Peter, a. a. O., S. 46.

28 Zu dieser Bewegung s. Liu Jen-Kai, ,,Zum Studium der Bewegung zur Unterdrückung der Konterrevolution von 1951 in der Volksrepublik China“, Oriens Extremus 1/2, Dez. 1977, S. 135-144.

29 Yangtse-Zeitung, Wuhan (changjiang ribao), 2. 6. 51; s. a. Glossary of Chinese Political Phrases (Union Research Institute, Hong Kong 1977), S. 250.

30 RMRB, 21. 8. 51, S. 2.

31 s. Tang, a. a. O., S. 379.
} 
Úbergang zum Sozialismus unternommen wurden. Der neue Vorsitzende Lai Ruoyu ging auf die Fehler der vergangenen Gewerkschaftsarbeit in privaten und staatlichen Betrieben ein. Er betonte, ,,daß die Führung des ACGB für kurze Zeit ökonomistische Fehler begangen hat. Diese Fehler zeigten sich in folgenden Aspekten: Bei der Gewerkschaftsarbeit in staatlichen Betrieben wurde die Betonung auf sogenannte Widersprüche zwischen öffentlichen und privaten Interessen gelegt mehr als auf die Identität zwischen den individuellen Interessen der Arbeiterklasse und den kollektiven, langfristigen Interessen der gesamten Arbeiterklasse, während die Arbeiterwohlfahrt als Widerspruch zur Entwicklung der Produktion angesehen wurde. Bei der Gewerkschaftsarbeit in Privatbetrieben lag die einseitige Betonung auf Beratungen zwischen Arbeitern und Kapitalisten. Hinsichtlich der Frage nach der Beziehung zwischen Gewerkschaft und Partei bestand die Tendenz, die Gewerkschaften von der Partei zu trennen. Außerdem bediente sich der ACGB bei der Behandlung verschiedener konkreter Probleme subjektiver Arbeitsmethoden, die in keinem Bezug zur Realität und zu den Massen standen. Diese Abweichungen führten jedoch zu keinem größeren Schaden, denn sie wurden vom Zentralkomitee der Kommunistischen Partei Chinas und vom Genossen Mao Zedong schnell entdeckt und korrigiert. Gleichzeitig war es auch so, daß viele Gewerkschaftsgruppen unter der Führung der lokalen Parteiorganisationen die falschen Anweisungen des ACGB gar nicht akzeptierten. Deshalb kann man sagen, daß die Gewerkschaft im Prozeß des Wiederaufbaus doch eine wichtige Rolle spielte."32 Die neue verabschiedete ,,Verfassung der Gewerkschaft" legte als wichtigste Aufgaben in der Zeit des nationalen Aufbaus fest: ,, unter der Führung der Kommunistischen Partei Chinas engen Kontakt mit den Arbeitern pflegen und sie erziehen, ihr Niveau des politischen Bewußtseins und der Organisation erhöhen, das Bündnis der Arbeiter und Bauern konsolidieren, sich mit Leuten aus allen Schichten zusammenschließen und aktiv den nationalen Aufbauplan erfüllen; dann, auf der Basis gesteigerter Produktion, Schritt für Schritt das materielle und kulturelle Leben der Arbeiterklasse und des ganzen werktätigen Volkes verbessern sowie für die systematische Realisierung der Industrialisierung und das Fortschreiten zum Sozialismus hin kämpfen. “33 Auf dem Gewerkschaftskongreß wurde auch der Kampf für die Einheit der Werktätigen aller Länder und die Verteidigung eines dauerhaften Friedens im Fernen Osten und der ganzen Welt betont. Staatspräsident Liu Shaoqi war einer der Vizevorsitzenden des Exekutivkomitees des kommunistischen Weltgewerkschaftsverbandes.

\section{Die Gewerkschaften bis zum 8. Nationalen Gewerkschaftskongreß 1957}

Die Jahre nach dem 7. Nationalen Gewerkschaftskongreß standen ganz im Zeichen der sozialistischen Umgestaltung der verschiedenen wirtschaftlichen Bereiche. Nach dem Ende der ,Drei-Anti- (gegen Unterschlagung, Verschwendung und Bürokratismus in den Staatsorganen) und Fünf-Anti-Bewegung“ erklärte Mao Zedong: ,,Die Zeit von der Gründung der Volksrepublik China bis zur praktischen Vollendung der sozialistischen Umgestaltung ist eine Ubergangsperiode. Die Generallinie und Hauptaufgaben währ end dieser Periode sind, über eine relativ lange Periode, die schrittweise Verwirklichung der sozialistischen Industrialisierung des Landes und die staatliche sozialistische Umgestaltung der Landwirtschaft, des Handwerks, der kapitalistischen Industrie und des kapitalistischen Handels. “34

\footnotetext{
32 Seventh All-China Congress of Trade Unions (Foreign Languages Press, Peking 1953), S. 52; zit. in: Peter, a. a. O., S. 64 f.

33 Eighth All-China Congress of the Trade Unions, a. a. O., S. 23.

34 GMRB, 22. 11. 53.
} 
Die Kommunistische Partei verfolgte gegenüber der nationalen Bourgeoisie eine sehr geschickte, gemäßigte und langfristige Politik. Sie schloß quasi mit ihr ein befristetes Bündnis, um sie sich zum Aufbau der nationalen Wirtschaft und zur Versorgung der ländlichen Bevölkerung mit Industriegütern zunutze zu machen. Was bedeutete staatliche sozialistische Umgestaltung? Für die Umwandlung Chinas von einem industriell unterentwickelten Land zu einem industriell blühenden Land,,ist es unerläßlich, die nicht sozialistische Industrie in die sozialistische Industrie zu überführen. Auf der einen Seite ist es notwendig, darum zu kämpfen, mehr vom Staat geleitete neue Fabriken und Bergwerke sozialistischer Natur zu bauen, mehr Eisen und Stahl zu schmelzen. . . Auf der anderen Seite ist es notwendig, die sozialistische Umgestaltung des Handwerks und der kapitalistischen Industrie durchzuführen, damit sie die Produktion in strikter Úbereinstimmung mit dem Staatsplan und den Bedürfnissen des ganzen Volkes durchführen können. “35 Dies war ein dringendes Gebot, sah doch das Jahr 1953 den Beginn von Chinas 1. Fünfjahresplan. Dieser Plan machte deutlich: „,Die Periode der ökonomischen Wiederherstellung unseres Landes ist im wesentlichen beendet, und die Periode des großangelegten, planmäßigen ökonomischen Aufbaus ist gekommen ${ }^{36}$." In den fünf Jahren gab der Staat 766400 Millionen yuan für Unternehmen im Bereich der Wirtschaft, der Kultur und Erziehung aus ${ }^{37}$. Die sozialistische Umgestaltung der vier wirtschaftlichen Bereiche sah Ministerpräsident Zhou Enlai auf der 4. Tagung des 1. Nationalen Volkskongresses (1957) im Hinblick auf das Privateigentum an Produktionsmitteln im Jahr 1956 als im wesentlichen beendet an. Der genossenschaftliche Zusammenschluß des Handwerks und der Landwirtschaft war erreicht, ebenso waren die privaten Betriebe der nationalen Bourgeoisie in gemischt staatlich-private Betriebe überführt worden (die der bürokratischen Kapitalisten waren gleich nach der Befreiung enteignet worden), wobei den Kapitalisten Festzinsen von fünf Prozent ihres Kapitalanteils unabhängig vom Produktionsertrag gezahlt wurden.

Die mit dem 1. Fünfjahresplan einsetzende forcierte Industrialisierung hatte auch einen Wandel in der Gewerkschaftsstruktur zur Folge. Kurz nach der Befreiung waren lokale Gewerkschaftsräte bestimmend gewesen, nun wurde der Aufbau national organisierter Industriegewerkschaften gefördert. Das bedeutete, daß alle Gewerkschaftsmitglieder eines Unternehmens oder einer Institution unabhängig von ihrer speziellen Tätigkeit zu einer einzigen Basisgewerkschaft unter einer Industriegewerkschaft gehörten. Auf dem 7. Nationalen Gewerkschaftskongreß war die Wichtigkeit solcher Industriegewerkschaften betont worden. Die oben angesprochenen Basisgewerkschaften, die sich in Fabriken, Bergwerken oder Institutionen mit mehr als zehn Mitgliedern bilden konnten, sollten neben der Mobilisierung zur Produktion ,, unter der Führung der Partei und der oberen Ebenen der Gewerkschaften die breiten Massen der Arbeiter erziehen und organisieren, ihr Klassenbewußtsein und technisches Niveau erhöhen, neue Arbeitseinstellungen ermutigen, Arbeitsbeziehungen organisieren, die Erfüllung des nationalen Plans sichern und zugleich aktiv der Verbesserung der Arbeits- und Lebensbedingungen der Arbeiter und dem Schutz des täglichen Wohlergehens der Arbeiter Aufmerksamkeit schenken“"38.

Als Hauptaufgabe der Gewerkschaften im Sozialismus wird u. a. von Mao Zedong die Entwicklung der Produktion angesehen ${ }^{39}$. Die Erreichung dessen beinhaltet allerdings einen

\footnotetext{
35 Leitartikel der RMRB vom 9. 11. 53.

36 Zhou Enlai, s. RMRB, 28. 12. 52, S. 1.

37 s. Leitartikel der RMRB vom 6 1. 58.

38 JFRB, 12. 9. 51; s. a. P. Harper, ,Trade Union Cultivation of Workers for Leadership“, in: John W. Lewis (Hrsg.), The City in Communist China (Stanford, California 1971), S. 126.

39 s. H. Martin (Hrsg.), MaoTse-tung, Das machen wir anders als Moskau! Kritik an der sow jetischen Politökonomie (Hamburg 1975), S. 48 .
} 
ganzen Komplex von Maßnahmen auf seiten der Gewerkschaften, wie die Organisierung von sozialistischen Arbeitswettbewerben, die Hebung des kulturellen Niveaus der Arbeiter durch Beseitigung des Analphabetentums als ersten Schritt, die Hebung des technischen Niveaus durch das Lernen moderner Produktionstechniken, etc. Das Problem zum Zeitpunkt der Befreiung bestand neben der geringen zahlenmäßigen Stärke der Arbeiter in ihrem niedrigen kulturellen Niveau, was bewußte Änderungen in der Einstellung zu moderner Technik zwecks Úbernahme neuer Technologien zu einer schweren Aufgabe werden ließ. 1949 gab es nur 3000000 Industriearbeiter, und die Gesamtzahl der Arbeiter und Angestellten betrug $8000000^{40}$. In der Arbeiterklasse soll es damals 75 bis 80 Prozent Analphabeten gegeben haben. Als dringendstes Problem für die Gewerkschaften nach der Befreiung jeder einzelnen Stadt in China wurde die Erhöhung des politischen Bewußtseins angesehen, das heißt vor allem, die Arbeiter sich der Aufgabe bewußt werden zu lassen, die Führung im Land zu übernehmen. Die Gewerkschaften spielten nun eine große Rolle in der politischen Erziehung und auch bei der Organisierung von Freizeit-Bildung. Freizeit-Schulen wurden als ein geeignetes Mittel zur Erhöhung des Wissens der Werktätigen angesehen. Der Verwaltungsrat der Zentralen Volksregierung erließ deshalb am 1. 6. 1950 die ,,Direktiven zur Entwicklung der Freizeit-Bildung für Arbeiter und Angestellte“41, die festlegten, daß die Gewerkschaftsorganisationen in den Betrieben für die Ausführung der Freizeit-Bildung der Arbeiter und Angestellten verantwortlich seien. 60 Prozent der Geldmittel für Kultur und Erziehung, die der Betrieb den Gewerkschaftsorganisationen zukommen ließ, sollten für die Freizeit-Bildung verwendet werden. 1950 besuchten 760000 Arbeiter Freizeit-Klassen ${ }^{42}$. Lai Ruoyu konnte auf dem 7. Nationalen Gewerkschaftskongreß feststellen, daß an 16277 FreizeitSchulen 3087000 Arbeiter studierten und 535000 lesen und schreiben lernten. Die Teilnehmerzahl an technischer Aus- und Weiterbildung betrug 477 000. Zur Steigerung des Wissensstandes der Arbeiter trug auch der gewerkschaftseigene Arbeiter-Verlag bei, der allein zwischen September 1949 und März 1953339 Büchertitel und Zeitschriften in einer Auflage von 35574000 herausgab ${ }^{43} .1954$ nahmen von 18809000 Arbeitern und Angestellten über 3000000 an den verschiedenen, von der Gewerkschaft organisierten Freizeit-Bildungskursen teil. 953000 Arbeiter studierten in technischen Klassen, davon betrieben 100000 ein volles technisches Studium ${ }^{44}$. Im Dezember 1955 fand eine Nationale Arbeiterkonferenz über Freizeit-Bildung für Industriearbeiter statt, die sich mit den Hauptproblemen beschäftigte, wie schleppende Einrichtung eines umfassenden Schulangebotes, wenig Zeit zum Studium, wenig qualifizierte Lehrer und Mangel an Geldern. Vermehrte Anstrengungen in diesen Problemgebieten wurden unternommen, so daß 1956 viele Betriebsanlagen über Klassen von der Grundschule bis zum Universitätsrang verfügten. Der Anteil der Analphabeten unter den Arbeitern und Angestellten war inzwischen merklich gesunken, 1957 betrug er nur noch 20,8 Prozent ${ }^{45}$.

Den Gewerkschaften oblag auch die Aufsicht über die Einhaltung des Arbeitsschutzes. Die Zahl der tödlichen Unfälle war 1952 im Vergleich zu 1949 um 63 Prozent gesunken, die der Verletzten im gleichen Zeitraum um 48 Prozent ${ }^{46}$. Auch für die Verwaltung der Arbeitsversicherung war die Gewerkschaft zuständig. Die am 26. 2. 1951 erlassenen ,,Arbeitsversiche-

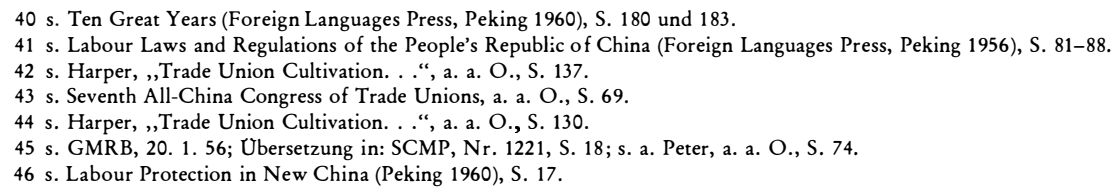


rungsbestimmungen der Volksrepublik China" sahen vor, daß in staatlichen, gemischt staatlich-privaten oder kooperativ geführten Betrieben mit über 100 Arbeitern und Angestellten die Leitung der Versicherung drei Prozent der totalen Lohnsumme zahlen mußte ${ }^{47}$. Die Arbeitsversicherung half im Altersfall, bei Unfällen und bei Krankheit. 1954 standen durch dieses System 126 Krankenhäuser mit 13000 Betten, 15 Altersheime, 72 Erholungsheime, 1400 Fabrikhospitäler mit 24 Betten im Durchschnitt, sechs Waisenhäuser, 4003 Kinderstuben für 120000 Kinder im Jahr und 2600 Säuglingszimmer mit einer Aufnahmekapazität von 37000 Babys zur Verfügung ${ }^{48}$. Für das Angebot dieser sozialen Maßnahmen wie auch der kulturellen und sportlichen Freizeitmöglichkeiten zahlte ein Gewerkschaftsmitglied nur ein Prozent seines Monatslohns als Beitrag.

Diese gewerkschaftlichen Aktivitäten für das Wohl der Arbeiter ermöglichen es, daß diese sich, sozial abgesichert, in Ruhe der Produktionstätigkeit widmen können. Auf dieser Grundlage haben nun die Gewerkschaften in erster Linie für eine Produktionssteigerung zu sorgen. Hauptmittel dazu ist der sozialistische Arbeitswettbewerb, durch den herausragende und ,Modellarbeiter“ gewonnen werden, die dann auch leitende Funktionen übernehmen können. Diese Art des Wettkampfes, der in Fabriken, zwischen Fabriken desselben Ortes oder verschiedener Orte stattfindet, nimmt zuweilen den Charakter einer Kampagne an, ebenso wie der Aufruf an die Arbeiter, Rationalisierungsvorschläge zu machen. 1950 sollen 600000 Arbeiter $^{49}, 19512380000^{50}$ und 1952 sogar 80 Prozent $^{51}$ an Produktionswettbewerben teilgenommen und 1952980000 Vorschläge gemacht haben ${ }^{52}$. Für Modellarbeiter finden sogar eigene Nationale Kongresse statt. Diesen Arbeitern kommt die Aufgabe zu, nicht so Fortgeschrittene auf ein höheres Niveau zu bringen. Die Gewerkschaften haben diesen Wissensaustausch, ,, mit den Fortschrittlichen wetteifern, von ihnen lernen und sie einholen sowie den Zurückgebliebenen helfen", anzuleiten und zu fördern.

Gemäß der marxistisch-leninistischen Lehre, daß die Gewerkschaft neben einer Schule des Kommunismus auch eine der Wirtschaftsführung (Produktionsplanung, Produktionsleitung, Arbeitsorganisation etc.) und Verwaltung ist, sind die Gewerkschaften verpflichtet, Arbeiter schrittweise an die Verwaltung und Leitung der Betriebe heranzuführen. Aktive Mitarbeit in Gewerkschaften ist eine gute Voraussetzung für die Übernahme von leitenden Posten.

Fehler und Nachlässigkeiten, die in den oben aufgeführten Aufgaben der Gewerkschaften auftraten, resultierten meist aus dem in der Praxis nur ungenügend gelösten Problem des Verhältnisses von Gewerkschaft und Partei. 1955 erschien in der Halbmonatszeitschrift „,Studium“ (xuexi) ein Artikel ,,Uber die Frage der Führung der Gewerkschaft durch die Partei“, der zeigte, daß die Krise von 1951 in der Gewerkschaftsarbeit noch nicht überwunden war. ,,Die Partei ist die Avantgarde der Arbeiterklasse, die Gewerkschaft ist die umfassendste Massenorganisation der Arbeiterklasse, folglich müssen die Arbeit der Partei und der Gewerkschaft unterschiedlich sein. . . Aber in Wirklichkeit gibt es viele Gewerkschaftskader, die in der praktischen Arbeit Partei und Gewerkschaft miteinander vermischen. Das Resultat ist, daß einige Parteimitglieder, die gewerkschaftliche Arbeit machen, die Funktion der Gewerkschaftsorganisation nicht begreifen. Sie sind der Ansicht, daß die Gewerkschaftsarbeit eine Zusatzbelastung darstellt, und führen deshalb die Gewerkschaftsarbeit

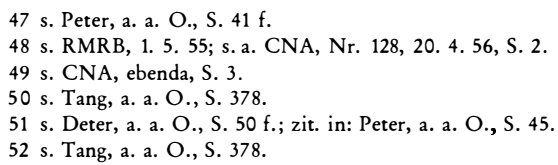


ohne innere Einsicht durch. Gleichzeitig führt es dazu, daß einige Gewerkschaftskader, die nicht Parteimitglieder sind, zu der Auffassung kommen, daß die Gewerkschaftsarbeit keinen Sinn hat, und sie deshalb vernachlässigen. " 53

Das Jahr 1957 erlebte im Rahmen der Hundert-Blumen-Bewegung eine Fülle von Aktivitäten auf dem Gebiet der Untersuchung der Gewerkschaftsarbeit. Untersuchungsgruppen stellten in den Städten Erhebungen über die Fehler an. Es erschienen zahlreiche Artikel in der ,Volkszeitung“ und der ,Arbeiterzeitung“, die auf die Krise in der Gewerkschaftsarbeit eingingen. Hauptresümee der Untersuchungen war, daß das Ansehen der Gewerkschaft unter den Arbeitern gesunken sei. Li Xiuren stellte fest, daß die Arbeiter auf die Gewerkschaft als „,Büro zur Verwaltung der Arbeiter“ herabsahen und sie bei Differenzen mit der Administration beiseite ließen. Als Parteimitglieder sei es für die Gewerkschaftsführer sehr schwierig, allzusehr für die Interessen der Arbeiter einzutreten, da sie sich sofort den Vorwurf des ,Syndikalismus“ zuzögen ${ }^{54}$. ,,Wir sind Parteimitglieder, und entsprechend der Parteidisziplin müssen wir uns den Beschlüssen der Parteiorganisation unterordnen, sonst werden wir möglicherweise als ,Syndikalisten', ,Agitatoren für die Unabhängigkeit von der Partei‘ oder als ,Schwanz der Massen' bezeichnet und vielleicht sogar aus der Partei ausgeschlossen. Andererseits sind wir von den Arbeitern gewählt, für ihre Interessen zu sprechen, und sollten uns dem Willen der Mehrheit der Arbeiter unterordnen, sonst werden wir von den Arbeitern als ,Schwanz der Administration' angeklagt und von ihnen beiseite gelassen. " 55 Manche Kader waren auch mit ihrem niedrigen Status unzufrieden und klagten, daß sie hinter den Partei-, Verwaltungskadern und Technikern nur auf Platz vier rangierten. Als Gewerkschaftskader genössen sie keinerlei Privilegien. Chen Yongwen traf in seinem in der „Arbeiterzeitung“ abgedruckten Bericht die Feststellung, daß die Gewerkschaften keine Macht hätten, die Probleme der Arbeiter zu lösen. ,,Heute ist das Prestige der Gewerkschaften nicht hoch; sie haben keinen Kontakt zu den Massen; diese merken, daß die Gewerkschaften ihre Probleme nicht lösen können. . . Wenn die Gewerkschaften wirklich die Interessen der Massen widerspiegeln wollen, ist eine gewisse Spannung zwischen den Gewerkschaften und der Verwaltung unvermeidlich."

Ein scharfer Vorwurf erging an Parteimitglieder, die ihre Gewerkschaftsarbeit nicht ernst nahmen; sie wurden der ,,drei nicht kommen“ beschuldigt: Sie kämen nicht zu Gewerkschaftstreffen, kämen nicht zur Berichterstattung und kämen nicht, um ihre Beiträge zu zahlen ${ }^{57}$. Am weitesten wagte sich der Chef der Archive des ACGB Gao Yuan vor. Er soll befürwortet haben, daß Arbeiter sich selbst organisieren sollten, wobei sie sich nicht unbedingt dem ACGB anzuschließen hätten. Diese Gewerkschaften könnten ihre Arbeit auch unabhängig von der Führung durch die Partei erledigen. Er beschuldigte die Gewerkschaften, sich nicht um das Wohl der Arbeiter und Angestellten zu kümmern. Die Arbeiter schlössen sich nicht den Gewerkschaften an, um den Marxismus zu studieren oder um beim ökonomischen Aufbau zu helfen, sondern allein zu ihrem eigenen Schutz. Die Schulung sei den Gewerkschaften von der Partei auferlegt, sie mache nicht das Wesen der Gewerkschaft aus. Dann räumte er ein, daß auch der Staat manchmal im Unrecht sei und die Interessen der Ar-

\footnotetext{
53 Xuexi 10/1955, S. 10 f.; zit. in: Peter, a. a. O., S. 77 t.

54 RMRB, 9. 5. 57; CNA, Nr. 183, 31. 5. 57, S. 3.

55 RMRB, 9. 5. 57; SCMP, Nr. 1551 (1957), S. 10-13.

56 GRRB, 30. 5. 57; CNA, Nr. 355, 13. 1. 61, S. 3

57 s. Harper, ,The Party and the Unions. . .", a. a. O., S. 111.
} 
beiter verletzen könne ${ }^{58}$. Auch der Gewerkschaftsvorsitzende Lai Ruoyu hatte in einem Interview zugeben müssen, daß die Gewerkschaften in der Vergangenheit nur Exekutivorgane der Verwaltung der Betriebe und deren Parteikomitees gewesen seien. Er betonte indes, daß die Gewerkschaft nicht jede Klage der Arbeiter unterstützen dürfe, denn ,,die Ansichten der Massen sind nicht immer richtig“ “59 Ein Leitartikel der „,Arbeiterzeitung“ vom November 1957 ging zusammenfassend auf die ganzen Schwächen der Gewerkschaftsarbeit ein. Er sprach vom Anwachsen der Forderungen und der Kritik der Massen, es gebe Klagen über Arbeitsorganisation, Löhne und Prämien, Arbeitsversicherung, Unterkunft und Lebensbedingungen allgemein. Die Massen hätten das Vertrauen in die Gewerkschaften verloren, so daß die Gewerkschaftsarbeit in einer Krise stecke. Das Hin- und Hergerissensein der Gewerkschaftskader zwischen den Interessen der Verwaltung und den Arbeiterinteressen verkörpere den grundlegenden Widerspruch zwischen den Interessen des Staates und denen des Volkes. Die Gewerkschaften müßten schon die Interessen der Massen vertreten, aber es dürfe doch eigentlich keinen Interessenkonflikt zwischen Staat und einzelnem Arbeiter geben, wo doch die Arbeiterklasse die führende Klasse im Staat sei. ${ }^{60}$ Der realiter existierende Konflikt lag in der Schwierigkeit, einerseits die täglichen Bedürfnisse einer 600-MillionenBevölkerung zu befriedigen und andererseits genug Kapital für den wirtschaftlichen Aufbau des Landes zu akkumulieren. All diese Probleme standen an, als der 8. Nationale Gewerkschaftskongreß im Dezember 1957 einberufen wurde.

\section{Der 8. Nationale Gewerkschaftskongreß 1957}

Der 8. Nationale Gewerkschaftskongreß fand schon in der neuen historischen Periode des Sozialismus statt. Die sozialistische Umgestaltung der Landwirtschaft, des Handwerks, der kapitalistischen Industrie und des kapitalistischen Handels war im wesentlichen abgeschlossen, damit erlebte die Zeit der Neuen Demokratie ihr Ende. " Gleich die ersten Jahre, 1956 und 1957, erlebten Kampagnen großen Ausmaßes. „Laßt hundert Blumen nebeneinander blühen! Laßt hundert Schulen miteinander wettstreiten!“', so hatte es Mao Zedong gefordert, das Blühen für Literatur und Kunst, den Wettstreit für die Wissenschaft. ,,Große Aussprache" und ,,große Meinungsäußerung" setzten nun in den verschiedenen Kreisen ein, ,,große Debatten“ und Wandzeitungen wurden propagiert. Dem ,Blühen und Wettstreit" folgte 1957 der Kampf gegen die Rechten, das heißt gegen ,,bürgerliche Rechte“, die die Hundert-Blumen-Bewegung auf den Bereich der Politik ausdehnen und für ihre eigenen Ziele ausnutzen wollten sowie das politische System als Ganzes in Frage stellten und angriffen. Der Kampf erstreckte sich auch auf die Gewerkschaften, so wurde u. a. Gao Yuan als „Rechter“ eingestuft. 1958 traf Chen Yongwen das gleiche Schicksal. Im September 1957 begann ferner eine Ausrichtungsbewegung in den Unternehmen durch sozialistische Erziehung der Arbeiter. Im November sah Lai Ruoyu für die Bewegung das Stadium der Reformvorschläge gekommen. Er rief die Arbeiter auf, nicht mit ihrer Meinung zurückzuhalten aus Angst, als ,,Rechte“ abgestempelt zu werden. ,,Solche Sorgen sind unnötig. Unser freier Ausdruck der Gedanken betrifft das Studium unserer Arbeitsmethoden mit dem Ziel, sie zu

\footnotetext{
58 s. GRRB, 11. 11. 57; CNA, Nr. 207, 29. 11. 57, S. 2.

59 s. GRRB, 8. 5. 57 ; CNA, Nr. $183,31.5 .57$, S. 1 f.

60 s. GRRB, 12. 11. 57; s. a. CNA, Nr. 207, 29. 11. 57, S. 1.
} 
verbessern. “61 Wie alle Organisationen mußte auch der ACGB sein Personal auf zwei Drittel reduzieren, das eine Drittel ging zu körperlicher Arbeit in Fabriken und aufs Land. In dieser Situation traten nun die 989 Delegierten (davon 200 Modellarbeiter und herausragende Arbeiter) des 8. Nationalen Gewerkschaftskongresses zusammen, die 16300000 Mitglieder repräsentierten ${ }^{\mathbf{6 2}}$. Auf diesen Kongreß soll hier ausführlich eingegangen werden, da auf ihn eine Pause von 21 (!) Jahren folgte; so fand der 9. Nationale Gewerkschaftskongreß nicht - wie festgelegt - fünf Jahre später, 1962, statt, sondern er wird im Oktober 1978 abgehalten werden.

In seinem Rechenschaftsbericht ${ }^{63}$ zeigte Lai Ruoyu zunächst die Erfolge in der Gewerkschaftsarbeit der vergangenen Jahre auf: Seit dem 7. Kongreß seien 2600000 herausragende Arbeiter und Angestellte hervorgetreten und 4560000 Rationalisierungsvorschläge gemacht worden. Mehr als 5000000 Arbeiter und Angestellte würden zum Zeitpunkt des 8 . Kongresses a n allgemeinen Bildungskursen im Freizeitprogramm teilnehmen. Auch bei der Reform des Lohnsystems und bei Lohnerhöhungen hätten die Gewerkschaften mitgeholfen. So sei das Lohnniveau der Arbeiter und Angestellten 195637 Prozent höher als 1952. Für den Arbeitsschutz würden die Gewerkschaften ebenfalls Sorge tragen; dafür habe der Staat in den letzten fünf Jahren 290000000 yuan ausgegeben. Was soziale Einrichtungen angehe, so könnten die Gewerkschaften in den von ihnen errichteten Sanatorien 25000 Betten vorweisen; Basisgewerkschaftsorganisationen hätten 8200 Säuglingsstationen errichtet. Die Organisationsarbeit der Gewerkschaften erlebe einen großen Fortschritt, so daß unter den 16300000 Mitgliedern immerhin 3890000 Aktivisten seien. Als Hauptaufgabe für die nächsten Jahre nannte Lai die Verwirklichung des 2. Fünfjahresplanes. Wichtigste Frage sei die Durchführung der Massenlinie der Partei und der enge Kontakt mit den Massen. Anschließend gab Lai einen Úberblick über die Betriebsstrukturen. Grundlegende Politik für deren Leitung sei der demokratische Zentralismus, das heißt Demokratie unter zentralisierter Leitung, die Koordination einer einheitlichen Führung mit der Massenlinie. ,,In den ersten Jahren nach der Befreiung wurden Fabrik-Leitungskomitees und Arbeiterdelegierten-Konferenzen in Staatsbetrieben errichtet. Konsultativkomitees von Arbeit und Kapital und Komitees, die helfen, die Produktion zu steigern und die Praktik der Wirtschaftlichkeit zu pflegen, wurden in privaten Betrieben organisiert, genau wie Komitees für demokratische Leitung in gemischt staatlich-privaten Betrieben. “ Nach dem 8. Parteitag der KPCh 1956 sei das System der Arbeiterkongresse eingeführt worden, die größere und klarer definierte demokratische Rechte als die Arbeiterdelegierten-Konferenzen hätten. Als Basisorganisation dieser Kongresse dienten die kleinen Gewerkschaftsgruppen. Rückblickend kritisierte Lai Ruoyu die Fehler in der Gewerkschaftsarbeit. Vor 1952 habe man zeitweilig zu einseitig die Frage des Lebensunterhaltes der Massen betont und dabei die Bedeutung der Produktion übersehen. Danach sei der umgekehrte Fehler eingetreten. Auch bei der Lohnreform 1956 habe es einige Ungerechtigkeiten gegeben. Bei Wohlfahrtsproblemen sei allzu schnell Hilfe vom Staat angefordert worden. Es gehe aber darum, die Arbeiter zur selbständigen Lösung von Problemen zu mobilisieren, das gelte sogar für den Wohnungsbau. Ein weiteres Problem sei die Klassenzusammensetzung der Arbeiter. Von den 13000000 Industriearbeitern und -angestellten 1957 seien nur 32 Prozent vor der Befreiung schon Arbeiter gewesen. Von den 68 Prozent der neuen Arbeiter stamme über die Hälfte aus Bauernfamilien oder habe vorher zu Studenten und Armen der Stadt gehört; drei Prozent der neuen Arbeiter stammten

61 GRRB, 9. 11. 57; CNA, Nr. 355, 13. 1. 61, S. 6.

62 s. Eighth All-China Congress of the Trade Unions, a. a. O., S. 8 und 27.

63 s. Eighth All-China Congress of the Trade Unions, a. a. O., S. 21-64. 
aus Familien von Grundherren, reichen Bauern und Kapitalisten, kämen von der Polizei und den Armeeinheiten der Guomindang Tschiang Kai-schek's oder seien Müßiggänger gewesen. Zur Verhinderung des Einflusses kleinbürgerlicher Ideen müsse das politische Bewußtsein der Arbeiter erhöht und eine kleine Zahl aus Ausbeuterklassen ideologisch umerzogen werden. Lai Ruoyu ging dann auf das Verhältnis zur Partei und die Beziehung zwischen Produktionssteigerung und Sorge für das Wohl der Arbeiter ein. ,Seit der Befreiung hielten Gewerkschaftskader zwei wichtige Debatten über die grundlegende Politik ab, der die Gewerkschaftsarbeit folgen sollte. Die erste fand statt, als es Meinungsunterschiede unter dem führenden Gewerkschaftspersonal über die Politik und Aufgaben der Gewerkschaft nach der Vollendung der demokratischen Revolution und der Errichtung eines volksdemokratischen Staates, geführt von der Arbeiterklasse, gab. Diese Frage wurde zwischen 1951 und 1953 gelöst, als der 7. Nationale Gewerkschaftskongreß abgehalten wurde. Die zweite Debatte betraf die Zweifel und das Schwanken gewisser Genossen hinsichtlich der grundlegenden Politik, die die Gewerkschaftsarbeit und Aufgaben in der Periode des nationalen Aufbaus leitete, als die sozialistische Revolution den grundlegenden Sieg in der Frage des Eigentums an Produktionsmitteln davontrug. Dies wurde während der jüngsten Ausrichtungsbewegung gelöst. Zwei Fragen waren beiden Debatten gemeinsam: Erstens, ob von den Gewerkschaften verlangt wurde, die Führung der Partei zu akzeptieren; zweitens, ob die Gewerkschaften die Produktion als ihre zentrale Aufgabe ansehen sollten und wie man korrekt die Beziehung zwischen der Entwicklung der Produktion und der Verbesserung des Lebensunterhaltes der Arbeiter behandelt. "Lai’s Fazit war, daß sich bei Befolgung der korrekten Linie der Partei für die Gewerkschaftsarbeit Erfolge einstellten. ,,Aber immer wenn eine Gewerkschaft von der Führung durch die Partei und ihrer Politik abwich, die Notwendigkeit, die Produktion zu steigern, ignorierte und sich allein auf Verbesserungen im Lebensunterhalt der Arbeiter konzentrierte, die Widersprüche zwischen Gewerkschaft und Verwaltung überbetonte und das Schmieden der Einheit vernachlässigte, . . . fiel ihre Arbeit in den Sumpf von Ókonomismus und Syndikalismus, und sie trennte sich von den Massen." Betont wurde ferner, daß die Gewerkschaftsarbeit in lokalen Betrieben unbedingt unter der Führung der betreffenden Parteikomitees zu stehen habe.

Wichtige Entscheidungen wurden auf dem 8. Nationalen Gewerkschaftskongreß auf dem Gebiet der Organisation getroffen. War seit 1953 die vertikale Struktur betont worden (Organisation nach Industriegruppen), kam jetzt die horizontale Struktur, das heißt die Organisation nach lokalen Gesichtspunkten, hinzu. Es herrschte also nun eine Zweigleisigkeit in der Gewerkschaftsorganisation. Dazu sagte die in einigenPunkten geänderte, neue Gewerkschaftsverfassung ${ }^{64}$ : ,,Die Gewerkschaften in China sind nach dem Prinzip der Koordinierung der Organisation nach Industriegruppen mit der Organisation nach lokalen Gebietskriterien gebildet. “ Ersteres bedeutet, daß ,,alle Gewerkschaftsmitglieder im selben Unternehmen, in der selben Institution oder Schule in einer einzigen Basisorganisation organisiert sein sollen“. „,Gewerkschaftsmitglieder im selben Industriezweig oder in ähnlichen Zweigen der nationalen Wirtschaft sollen in der selben nationalen oder lokalen Industriegewerkschaft organisiert sein. " Gewerkschaftsräte, auf verschiedenen Ebenen organisiert, ,,sollen die gemeinsamen führenden Organe ihrer untergeordneten lokalen Gewerkschaftsorganisationen und Industriegewerkschaftsorganisationen in ihrem Gebiet sein. Der Allchinesische Gewerkschaftsbund ist das nationale, gemeinsame, führende Organ aller lokalen Gewerkschaftsräte und aller nationalen Industriegewerkschaftsorganisationen." (Art. 9) Daß die

64 s. ebenda, S. 106-125. 
Zweigleisigkeit der Gewerkschaftsorganisation erhebliche Verwirrung mit sich brachte, gestand Xu Zhizhen in seinem Bericht zur Revision der Gewerkschaftsverfassung ein: ,Einige Genossen haben verlangt, daß der Verfassungsentwurf genaue Vorstellungen über die Funktionen und Verantwortlichkeiten der lokalen Gewerkschaftsräte und der lokalen Zweige der Industriegewerkschaften enthalten solle. Wir finden, daß dies noch nicht getan werden kann, weil die Frage ziemlich kompliziert ist und wir in dieser Beziehung nicht genug Erfahrung haben. "65 Die neue Verfassung legte in ihrem allgemeinen Programm ${ }^{66}$ den Aufbau des Landes in einen starken sozialistischen Staat als Hauptaufgabe der nächsten Jahre für die Arbeiter fest. Zum Verhältnis Gewerkschaft und Partei sowie zu der Beziehung zwischen den Staatsinteressen und den individuellen Interessen hieß es an gleicher Stelle ganz eindeutig: „,Die Gewerkschaften in China sind die Massenorganisationen der Arbeiterklasse, geführt von der Kommunistischen Partei Chinas, und sind die Transmissionsriemen zwischen Partei und Massen. Unter der demokratischen Diktatur des Volkes sind die Gewerkschaften eine Schule der Verwaltung, eine Schule der Leitung und eine Schule des Kommunismus für die Arbeiter.“ „Die Gewerkschaften sind die stärkste gesellschaftliche Stütze unserer demokratischen Diktatur des Volkes. “,,Unter der Führung der Arbeiterklasse sind die Interessen des Staates mit den allgemeinen Interessen des ganzen Volkes und auch mit den fundamentalen Interessen unserer Arbeiterklasse identisch. Die Gewerkschaften sollten die Arbeiter dazu erziehen, die Einheit der Interessen von Staat und Individuen zu erkennen und, wenn diese beiden in Konflikt geraten, zu erkennen, daß die individuellen Interessen denen des Staates untergeordnet sein sollten."

In den noch existierenden gemischt staatlich-privaten Betrieben kam den Gewerkschaften ferner gemäß dem allgemeinen Programm der Verfassung die Aufgabe zu, diese Betriebe und die Kapitalisten umzuformen, so daß letztere schließlich ihren Lebensunterhalt durch eigene Arbeit verdienen könnten.

Die Verfassung bestand aus 39 Artikeln: I Mitgliedschaft (Art. 1-7), II Organisationsstruktur (Art. 8-14), III Das höchste nationale führende Organ der Gewerkschaften (Art. 15-21), IV Lokale Gewerkschaftsorganisationen (Art. 22-27), V Basisgewerkschaftsorganisationen (Art. 28-34), VI Fonds (Art. 35-36) und VII Anhang (Art. 37-39). Der Allchinesische Gewerkschaftsbund sollte von nun an alle fünf Jahre einberufen werden (vorher alle vier Jahre) in Einklang mit dem Fünfjahresplan. Zwischen den Gewerkschaftskongressen nimmt das Exekutivkomitee, das zweimal im Jahr zu einer Plenartagung zusammentreten soll, die Aufgaben wahr; es wählt ein Präsidium und ein Sekretariat; viermal im Jahr soll eine Plenarsitzung des Präsidiums stattfinden. Die Nationalen Kongresse einer Industriegewerkschaft sollen alle drei oder vier Jahre vom Nationalen Komitee der Gewerkschaft einberufen werden, das in der Zwischenzeit für die Durchführung deren Entscheidungen und der Entscheidungen des ACGB sorgt. Auf der regionalen Ebene nehmen die Komitees der lokalen Gewerkschaftsräte und die regionalen Industriegewerkschaftskomitees zwischen den Treffen ihrer Kongresse die Aufgaben wahr. An der Basis der Gewerkschaften stehen die Basisorganisationen. In Betrieben, Institutionen oder Schulen mit zehn und mehr Mitgliedern kann ein Gewerkschaftskomitee errichtet werden, das zwischen den allgemeinen Mitgliedertreffen oder Delegiertentreffen tätig ist. Arbeiterkongresse in Fabriken und Bergwerken stellen die Organisation dar, durch die die Teilnahme der Werktätigen an der Leitung, die Úberwachung der Verwaltung und die Verbesserung der Selbsterziehung ermöglicht werden. Das Basisgewerkschaftskomitee setzt Werkstatt- oder Abteilungskomitees ein. Unter den Basis-

65 s. ebenda, S. 99.

66 s. ebenda, S. 106-110. 
gewerkschaften oder den letzteren Komitees können Gewerkschaftsgruppen gemäß den Produktions- oder Arbeitseinheiten gebildet werden. Die Hauptaufgaben der Basisgewerkschaften werden wie folgt definiert: Organisierung der sozialistischen Wettbewerbskampagnen, Austausch fortschrittlicher Arbeitserfahrungen, Ansporn zu Rationalisierungsvorschlägen, Stärkung der Arbeitsdisziplin, Garantierung der Erfüllung der Aufgaben des nationalen Aufbaus, Stärkung der politischen und ideologischen Erziehung, Erziehung der Arbeiter im Geist des Kommunismus, Förderung von Kritik und Selbstkritik; Uberwachung der Verwaltung bei der Angleichung von Löhnen, Verbesserung des Arbeitsschutzes, von Lebens- und Wohnbedingungen, des kulturellen und Wohlfahrtsangebots und bei der vollen Erfüllung der Politik, der Gesetze und Bestimmungen des Staates sowie der Entscheidungen der Arbeiterkongresse; Bemühung um Verbesserung des kulturellen und materiellen Lebens sowie der Arbeitsbedingungen der Arbeiter, Sorge für die Arbeitsversicherung, Förderung von kulturellen, Erholungs- und Sportaktivitäten, Unterstützung der Verwaltung bei der Organisierung des kulturellen und technischen Studiums der Arbeiter, Heranziehung von Aktivisten, Gewinnung neuer Mitglieder, Einsammeln der Mitgliederbeiträge sowie Prüfung der Zuwendungen, die die Verwaltung an den Gewerkschaftsfonds und für die Arbeitsversicherung zahlt, etc. Der Gewerkschaftsfonds speist sich aus folgenden Quellen: 1) Mitgliedsbeitrag, der ein Prozent des Monatslohns beträgt; 2) Aufnahmegebühr neuer Mitglieder, die ebenfalls ein Prozent des Lohns ausmacht; 3) Einnahmen aus Sport- und kulturellen Veranstaltungen; 4) Zuwendungen der Verwaltung.

Der 8. Nationale Gewerkschaftskongreß hatte zum einen die Führung der Partei über die Gewerkschaft, zum anderen die Basis der Gewerkschaften gestärkt. Das Gewicht der Partei kam schon darin zum Ausdruck, daß von den 989 Delegierten 91 Prozent Parteimitglieder waren. Der Anteil der Parteimitglieder an der Basis hatte sich von 6,3 Prozent im Jahr 1951 auf 14 Prozent im Jahr 1956 erhöht ${ }^{67}$. 1956, auf dem 8. Parteitag der KPCh, hatte LiXuefeng folgende Zahlen genannt: ,, . . die Zahl der Parteimitglieder in Betrieben hat 10 bis 20 Prozent der Gesamtzahl von Arbeitern und Angestellten erreicht, während die meisten Kader Parteimitglieder sind." ${ }^{68}$

Lai Ruoyu wurde wieder zum Vorsitzenden des ACGB gewählt, er starb jedoch kurz darauf, und die 2. Plenartagung des Exekutivkomitees im August 1958 wählte Liu Ningyi, einen erfahrenen Mann der Arbeiterbewegung und Vizevorsitzenden bis 1957, zum Vorsitzenden.

\section{Die Gewerkschaften bis zu ihrer Auflösung in der Kulturrevolution}

Im Januar 1958 wurde auf der 1. Tagung des Exekutivkomitees des ACGB verkündet, daß die Gewerkschaften die Produktion fördern müßten, um ,,mehr, schneller, besser und wirtschaftlicher" den Sozialismus aufzubauen. Gleichzeitig müßten sie sich der Wohlfahrt, der Löhne, des Arbeitsschutzes und der Lebensbedingungen der Arbeiter verstärkt annehmen. ${ }^{69}$

Selbst nach dem 8. Nationalen Gewerkschaftskongreß gab es noch Gewerkschaftsführer, die sich über ihre genaue Rolle und die der Gewerkschaften nicht im klaren waren, weil infolge der Dominanz der Partei ja schließlich die Parteimitglieder alles erledigen könnten und sich

67 s. Martin/Bartke, a. a. O., S. 78.

68 s. Eighth National Congress of the Communist Party of China, Vol. II, (Foreign Languages Press, Peking 1956 ), S. 315.

69 s. CMRB, 18. 1. 58; CNA, Nr. 359, 10. 2. 61, S. 1. 
auch die Verwaltung um Fragen der Produktion und der Lebensbedingungen der Arbeiter kümmere. Zum 37. Jahrestag der Gründung der KPCh im Juli 1958 sah sich die Gewerkschaftszeitung genötigt, erneut ausführlich auf das Verhältnis zwischen Partei und Gewerkschaft sowie auf die Aufgaben der Gewerkschaftskader einzugehen. ,,Wenn die Gewerkschaftsorganisationen aller Ebenen sich nicht auf die Führung der Parteikomitees stützen, wenn sie vorgeben, die Führung der Parteikomitees zu akzeptieren, und doch für, den unabhängigen Charakter von Arbeiterorganisationen' plädieren, so bedeutet dies einen Bruch mit der Politik der Partei und Gleichgültigkeit gegenüber den grundlegenden Aufgaben der Partei; dieser Wunsch nach ,unabhängigen Gewerkschaftsaktivitäten“ ist ein Irrtum.“ „Der gute Gewerkschaftsarbeiter stellt sich auf die Seite des Proletariats; er akzeptiert die Forderungen der Arbeiter nicht unterschiedslos; er erklärt, was richtig ist, und kritisiert, was falsch ist; er erhöht das kommunistische Bewußtsein der Arbeiter und erfüllt die Politik der Partei; er erachtet nicht unangemessene Forderungen rückständiger Arbeiter als die Forderungen der gesamten Massen der Arbeiter; er rennt nicht hin und her, um die Partei und Regierung zu bitten, die Forderungen anzunehmen, noch ist er gegen die Vorherrschaft von Partei und Regierung über die Gewerkschaften, denn das wäre ein äußerst schwerwiegender Fehler."70

1958 wurden einige hohe Gewerkschaftsführer als ,,Rechte“ entlarvt. Neben dem schon genannten Chen Yongwen waren dies Du Cunxun, Vorsitzender der Gewerkschaft in der Provinz Hebei, Dong Ting, Mitglied des Sekretariats des ACGB, und Wang Rong, Vorstandsmitglied des ACGB. In der Verurteilung Du’s heißt es: ,Die Partei ist der höchste Repräsentant . . . der Arbeiterklasse. Die Ansicht, daß ,nur die Gewerkschaften sich um das Leben der Arbeiter kümmern', ist vollständig falsch. Die Hauptarbeit der Gewerkschaften muß zu allen Zeiten die Produktion sein. Sich um das Leben der Massen zu kümmern, ist Sache der 600-Millionen-Bevölkerung und sollte in völliger Úbereinstimmung mit der Politik der Partei durchgeführt werden. “71 Auch die anderen ,,Rechten“ wurden beschuldigt, die Sorge für die Interessen der Arbeiter nur vorzugeben und die Partei zu beleidigen, als ob sie nicht die Interessen der Arbeiter berücksichtigte.

Das Jahr 1958 sah enorme Umwälzungen in China, den Großen Sprung nach vorn, der auf eine schnelle und große Produktionssteigerung in Landwirtschaft und Industrie abzielte, und die Einführung der Volkskommunen, die über verschiedene Stadien der Organisation der Bauern sorgsam vorbereitet war. Die folgenden Jahre standen nun unter der Generallinie „Unter Anspannung aller Kräfte immer vorwärtsstreben, um mehr, schneller, besser und wirtschaftlicher den Sozialismus aufzubauen". Mit dem Großen Sprung nach vorn erlebte auch die kulturelle und technische Erziehung der Arbeiter in Freizeit-Kursen, -Schulen und -Universitäten einen neuen Aufschwung. Es war schon ein Fortschritt, daß 1959 die meisten Arbeiter das Bildungsniveau der Grundschule hatten. ${ }^{72}$ Etwa 9000000 Arbeiter hatten das Niveau der Mittelschule erreicht, und über 400000 besaßen Hochschulniveau. ${ }^{73}$ Mitte 1959 gab es 444 bzw. 608 technische Schulen mit 190000 bzw. 270000 Studierenden. Ende 1959

\footnotetext{
70 Leitartikel der GRRB, 1. 7. 58; CNA, ebenda, S. 3 f.

71 GRRB, 3. 7. 58; CNA, ebenda, S. 4.

72 s. Leitartikel der RMRB, 29. 12. 58, S. 7.

73 s. NCNA, 25. 12. 59; SCMP, Nr. 2167, S. 17
} 
war die Zahl dieser Schulen auf 744 angestiegen, die Zahl der Studierenden betrug $280000 .{ }^{\mathbf{7 4}}$ Zwischen 1959 und 1962 hatten infolge des Bildungssystems in der Industrie über 1000000 Arbeiter Grundschulklassen absolviert, 500000 die Unterstufe der Mittelschule, ca. 100000 die Oberstufe der Mittelschule und über 15000 die Freizeit-Universitäten. ${ }^{75} 1960$ sollen 25000000 Arbeiter in Freizeit-Klassen der Betriebe eingeschrieben gewesen sein. ${ }^{76}$

Die Gewerkschaftsstruktur erfuhr in diesen Jahren eine Änderung. 1958 hatte es noch 25 nationale Gewerkschaften gegeben, seit 1960 gab es nur noch $16 .{ }^{77}$ Auf der 4. Tagung des Exekutivkomitees des ACGB vom 19.11.-13. 12. 1962 fand auch ein Wechsel in der Führung statt, neuer Vizevorsitzender wurde Ma Chungu, ein Mann mit internationalen Gewerkschaftserfahrungen. Auf dieser Sitzung wurden auch die Hauptziele der Gewerkschaftsarbeit festgelegt: Erfüllung von Produktionssteigerung und Sparsamkeit, Förderung des sozialistischen Arbeitswettbewerbs, Hilfe für die Kollektivwirtschaft der Volkskommunen und die technische Erneuerung der Landwirtschaft. ${ }^{78}$

Im April 1965 jährte sich der 40. Gründungstag des ACGB. Zı diesem Anlaß definierte die „Arbeiterzeitung“ die Rolle der Gewerkschaften wie folgt: ,,Die Kommunistische Partei ist die Avantgarde der Arbeiterklasse, deshalb dient die Gewerkschaft der Partei und sollte nie von ihr abweichen. Das Gewerkschaftswesen ist keine reformistische Bewegung, es steht unter einer revolutionären Partei. Es gibt weder eine ,Unabhängigkeit der Gewerkschaften', wie sie in kapitalistischen Ländern verkündet wird, noch ist die Beziehung zwischen Partei und Gewerkschaft eine ,Allianz', wie die Revisionisten behaupten. . . Insoweit er mit dem Willen der Partei übereinstimmt, sollte der Willen der Massen berücksichtigt werden. Die Gewerkschaft sorgt sich um die materiellen und kulturellen Bedürfnisse der Arbeiter, aber sie wird dabei von ideologischen, politischen Erwägungen geleitet. "79 Die Gewerkschaften sollten es sich zu dieser Zeit zum Ziel machen, daß Betriebe und Arbeiter die ,,gute Qualifikation in fünf Beziehungen“ erreichten. Für die Betriebe bedeutete das: gute politische Arbeit, gute Erfüllung des Produktionsplans, gute Verwaltung, gute Organisierung des Alltagslebens der Arbeiter, guter Arbeitsstil der Kader; für den einzelnen Arbeiter: hohes politisches Bewußtsein, gute Erfüllung seiner Aufgaben, strikte Einhaltung der Arbeitsdisziplin, Ausdauer im Studium, Einträchtigkeit und Hilfsbereitschaft. Zur Heranführung der Arbeiter an moderne Techniken mobilisierten die Gewerkschaften ferner die Arbeiter zur Zusammenarbeit mit Technikern und Führungskadern, die sogenannte „Dreierverbindung. " 80

Vor Ausbruch der Kulturrevolution geriet die Gewerkschaft stark unter den Einfluß des Verteidigungsministers Lin Biao und seiner Linie. Sie übertrug die von Lin Biao und der Volksbefreiungsarmee propagierten Parolen auf die Gewerkschaftsarbeit. So wurden zum Beispiel die Erfahrungen der Armee in den ,,vier Dingen an erster Stelle“ studiert, das bedeutet, der Mensch kommt vor der Waffe, Politik vor anderer Arbeit, bei der politischen Arbeit steht die ideologische an erster Stelle, wobei Praxisbezogenheit vor Buchweisheit kommt. Lin Biao empfahl für die ganze Gesellschaft die schöpferische Anwendung der Mao-Zedong-Ideen, wobei er jedoch Mao's wegweisende Richtlinien aus dem Zusammenhang riß. In Verkennung der dialektischen Beziehung zwischen Revolution und Produktion

\footnotetext{
74 s. CNA, Nr. 361, 24. 2. 61, S. 6

75 s. RMRB, 26. 1. 64; SCMP, Nr. 3161, S. 8.

76 s. Leitartikel der RMRB, 3. 8. 60; SCMP, Nr. 2318, S. 7.

77 s. Harper, ,,The Party and the Unions. . “", a. a. O., S. 101

78 s. RMRB, 25.12. 62, S. 1.

79 GRRB, 30. 4. 65; CNA, Nr. 627, 2. 9. 66, S. 2

80 s. Peking Rundschau, Nr. 18, 4. 5. 65, S. 24
} 
- ähnlich wie bei der durch die Kulturrevolution hochgekommenen „Viererbande“ (Jiang Qing, Zhang Chunqiao, Yao Wenyuan und Wang Hongwen) - verkündete er: ,Die MaoZedong-Ideen müssen unter den Arbeitern und Bauern durchgeführt werden . . . und dann wird sich die geistige Kraft in eine große materielle Kraft verwandeln. . " ${ }^{81}$ Die Gewerkschaften machten sich diese Bewegung zu eigen, was jedoch auf einige Kritik in ihren Reihen stieß. So wurde zum Beispiel der Einwand gemacht: „,Die Parteikomitees leisten ideologische Arbeit. Wenn die Gewerkschaften dasselbe machen, wird dies dann nicht doppelte Anstrengung sein?““ oder ,Eine gute Leistung in der Produktion drückt die korrekten politischen Ideen aus. “ Diese vom Haupttrend abweichenden Ansichten wurden in der ,,Volkszeitung“ entschieden zurückgewiesen: ,,Politisches Denken muß eine Realität in der Produktion werden und die Erfüllung der Produktionsaufgaben garantieren. "82

In der Großen Proletarischen Kulturrevolution fanden die Gewerkschaften ein vorläufiges Ende. Der ACGB soll am 26. 12. 1966 aufgelöst worden sein, er wurde durch die ,ZZentrale Rebellengruppe Allchinesischer Roter Arbeiter“ ersetzt. Die „,Arbeiterzeitung“ stellte am 31. März 1967 ihr Erscheinen ein. Jiang Qing soll vor der Rebellengruppe den Gewerkschaftsbund als falsch, der Parteizentrale gegenüber ungehorsam und als von den Massen abgesondert verurteilt haben. ${ }^{83}$ Der Gewerkschaftsvorsitzende Liu Ningyi wurde 1967 auf Wandzeitungen angegriffen. Die Gewerkschaften erschienen den Roten Garden der Kulturrevolution wegen ihres Eintretens für die Steigerung der Produktion in schlechtem Licht, ferner wegen ihrer engen Verbindung mit dem Ehrenvorsitzenden des ACGB Liu Shaoqi, gegen dessen revisionistische Politik sich in der Kulturrevolution der 9. Linienkampf in der Geschichte der KPCh richtete und der gestürzt wurde. Es bildeten sich verschiedene Arbeiterorganisationen wie das ,Zentrale Gewerkschaftshauptquartier Großtruppe Revolutionäre Rebellen“ und das ,,Zentrale Gewerkschaftshauptquartier ,Der Osten ist rot', Revolutionäre Rebellentruppe“, die dann eine Vereinigung eingingen. In Shanghai, der Hochburg der ,,Viererbande“, entstand die „,Revolutionäre Rebellen-Generalgruppe der Arbeiter ,Der Osten ist rot". Nach 1969 wurde der Versuch unternommen, über die Einberufung von Arbeiterdelegierten-Kongressen neue Arbeiterorganisationen zu schaffen.

Im Juni 1968 erschien in der „,Peking Rundschau“ ein Artikel einer Gruppe „Proletarische Revolutionäre im ACGB“ über den Kampf zweier Linien in der Gewerkschaftsbewegung. Dort hieß es: , ,Gewerkschaften sind der Kern der Macht, mit dem die Partei die breiten Massen der Arbeiter und Angestellten um sich sammelt, um den Klassenfeind zu bekämpfen, sie sind große Schulen für das schöpferische Studium und die schöpferische Anwendung der Mao-Zedong-Ideen. “ Liu Shaoqi und seine Leute wurden attackiert, ,,Gewerkschaften für die Produktion“, ,,Wohlfahrtsgewerkschaften“ und ,Gewerkschaften des ganzen Volkes“ betrieben und Ókonomismus sowie Syndikalismus, der die Gewerkschaften über die Partei des Proletariats stellt und sie als die einzige Form der Führung in der Arbeiterbewegung ansieht, praktiziert zu haben. Schon früh sei Liu für die Restauration des Kapitalismus eingetreten, gleich nach der Befreiung habe er zur ,, Kooperation zwischen Arbeitern und Kapitalisten“ gedrängt und die Kapitalisten ,,für Jahrzehnte gedeihen“ lassen wollen. Nach der grundlegenden Vollendung der sozialistischen Umgestaltung habe er die falschen Parolen wie ,, friedliche Entwicklung" und ,,die Revolution ist im wesentlichen beendet" propagiert. Nun predige er, daß die ,,Technik an erster Stelle kommt“. Für Liu seien damals ,,die Widersprüche zwischen Proletariat und Bourgeoisie bereits gelöst“: ,,Die Kapitalisten haben

81 s. RMRB, 19.6. 66, S. 1.

82 s. RMRB, 14. 4. 66; s. a. CNA, Nr. 627, 2.9. 66, S. 3.

83 s. CNA, Nr. 674, 25. 8. 67, S. 2. 
ihr Eigentum ausgehändigt; sie sind keine Kapitalisten mehr." Der Klassenkampf gehe jedoch weiter, ,,die Gewerkschaften sind Instrumente des Klassenkampfes“. Seit der Befreiung hätte Liu in der Gewerkschaftsarbeit eine falsche Linie durchzusetzen versucht. Erst hätten die Revisionisten die Gewerkschaft in ein ,,unabhängiges Königreich“ verwandelt mit dem Ziel, die Partei durch die Gewerkschaften zu ersetzen, und dann nach massiver Kritik dieser Politik 1958 die Parole von den Gewerkschaften als ,,fügsamem Werkzeug“ der Partei vorgebracht, um die Gewerkschaften unter die Kontrolle der bürgerlichen Elemente in der KPCh zu bringen. ${ }^{84}$ Manches an dieser Kritik der ,,Proletarischen Revolutionäre“ erinnert stark an Positionen der ,Viererbande“.

Trotz der Tatsache, daß die alten Gewerkschaften durch Rebellenorganisationen weitgehend ausgeschaltet waren, haben sie noch ein gewisses Dasein geführt. So wurde im Februar 1969 eine ,,7. Mai“-Kaderschule der Gewerkschaft zur Umerziehung der Kader errichtet. ${ }^{85}$ Auch auf internationaler Ebene traten die Gewerkschaften noch auf. Im Frühjahr 1967 reisten Delegationen in den Kongo und nach Albanien. Im Sommer erließ der ACGB einen Protest gegen ,,die Verbrechen der faschistischen Autoritäten in Hongkong. “86

\section{Die Vorbereitungen zum 9. Nationalen Gewerkschaftskongreß 1978}

Nach langem Schweigen kündigte der Neujahrsleitartikel 1973 in der ,,Volkszeitung“ offiziell den beabsichtigten Wiederaufbau aller Massenorganisationen an: ,,Die Gewerkschaft, die Kommunistische Jugendliga, die Roten Garden, die Kleinen Roten Soldaten, die Organisation von armen Bauern und unteren Mittelbauern sowie die Frauenorganisationen sollen Schritt für Schritt konsolidiert werden."

Vom 16.-21. 4. 1973 fanden in Peking und Shanghai Gewerkschaftskongresse statt. Auf dem 6. Pekinger Gewerkschaftskongreß ${ }^{\mathbf{8 7}}$ waren Zhang Chunqiao und Yao Wenyuan, zwei Vertreter der ,,Viererbande“, anwesend, ferner Wu De als 1. Sekretär des Pekinger Parteikomitees. 2396 Delegierte nahmen teil, die meisten von ihnen waren Industriearbeiter, über ein Drittel Frauen. Wu De nannte als Hauptaufgabe der Gewerkschaft, unter der Führung der Partei die Arbeiter zu organisieren, damit sie den Marxismus-Leninismus und die Mao-Zedong-Ideen studierten, entschlossen am Klassenkampf und am Kampf zweier Linien festhielten sowie die Diktatur des Proletariats festigten. Während der Kulturrevolution hätten sich Pekings Industriearbeiter dadurch ausgezeichnet, daß sie in Antwort auf Mao Zedong's Ruf: ,,Die Arbeiterklasse muß in allem die Führung innehaben!“ seit dem 27. 7. 1968 in die Schulen und an andere Orte mit vielen Intellektuellen gegangen seien und so ,, Kampf-Kritik-Umgestaltung" (Kampf gegen die Machthaber, die den kapitalistischen Weg gehen; Kritik an den bürgerlichen, akademischen Autoritäten; Umgestaltung des Erziehungswesens und der Teile des Uberbaus, die nicht der sozialistischen ökonomischen Basis entsprechen) im Úberbau durchgeführt hätten. Uber 40000 Arbeiter waren in den letzten Jahren in die KPCh eingetreten, über 20000 neue Arbeiterkader herangereift. Das 6. Pekinger Gewerkschaftskomitee wurde gewählt, es hatte 105 Mitglieder und 15 Alternativmitglieder. 35 Pro-

\footnotetext{
84 Peking Review, Nr. 26, 28. 6. 68, S. 17-21.

85 s. NCNA, 1. 2. 69.

86 s. CNA, Nr. $674,25.8 .67$, S. 2.

87 s. NCNA, 23. 4. 73.
} 
zent waren Frauen. Das Ständige Komitee umfaßte 26 Mitglieder, Vorsitzender war Ni Zhifu.

Der 5. Shanghaier Gewerkschaftskongreß ${ }^{88}$ hatte 1598 Delegierte. Repräsentanten der Industriearbeiter machten 73 Prozent aus und die der Frauen 39 Prozent. Wang Hongwen, der junge Aufsteiger unter der ,,Viererbande“, der zum stellvertretenden Parteivorsitzenden ernannt wurde, hielt eine Rede. Auch die Shanghaier Arbeiter, so hieß es, hätten wie die Arbeiter in Peking eine große Rolle im Úberbau während der Kulturrevolution gespielt. Die Aufgaben derShanghaier Gewerkschaftsorganisation ähnelten denen der Pekinger Gewerkschaft. Das gewählte Gewerkschaftskomitee bestand aus 158 Mitgliedern; nie zuvor war ein Shanghaier Gewerkschaftskomitee so groß gewesen. 31 Prozent davon waren Frauen. Ein Ständiges Komitee wurde von der 1. Plenartagung des Komitees gewählt mit Wang Hongwen als Vorsitzendem.

Am 24. 4. 1973 feierte der Leitartikel in der ,,Volkszeitung“ - zu der Zeit waren die Medien von der ,Viererbande“ kontrolliert - die Sitzungen des Pekinger und Shanghaier Gewerkschaftskongresses als Startschuß für ähnliche Konferenzen auf Provinzebene sowie in größeren und mittleren Städten. Die Arbeiter in Shanghai hätten die Flamme der Revolution vom 27. Januar 1967 entzündet, während die Arbeiterklasse in Peking die Initiative im Entsenden von Repräsentanten ergriffen habe, die in Zusammenarbeit mit Soldaten der Volksbefreiungsarmee Propagandateams der Mao-Zedong-Ideen bildeten. In Anbetracht der Feststellung, daß es unter den zahlreichen Aufgaben der Gewerkschaft die fundamentale sei, während der gesamten historischen Periode des Sozialismus am Klassenkampf festzuhalten und für die sozialistische Revolution sowie für die Diktatur des Proletariats einzutreten, wurde Liu Shaoqi scharf angegriffen, der meinte, daß sich die Gewerkschaft nicht um den Klassenkampf zu kümmern brauche. Ziel der Anstrengungen müsse es sein, ,,das fortgeschrittene Weltniveau einzuholen und . . China in ein starkes sozialistisches Land zu verwandeln mit moderner Industrie, moderner Landwirtschaft, moderner Wissenschaft und Kultur sowie moderner Landesverteidigung“".

Im Jahr 1973 fanden die Gewerkschaftskongresse auf Provinzebene, das heißt in den Provinzen, regierungsunmittelbaren Städten (Peking, Shanghai und Tientsin) und Autonomen Gebieten, statt mit Ausnahme von Ningxia, Fujian und Sichuan. Gewerkschaftskongresse des Autonomen Gebietes Guangxi, der Provinz Yunnan und der Provinz Hubei wurden vom 20.-27. 6. abgehalten, auf denen neue Führungsorgane gewählt wurden. An jedem Kongreß nahmen über 1000 Delegierte teil, über 60 Prozent waren Industriearbeiter, etwa 30 Prozent Frauen. Uber 70000 herausragende Arbeiter dieser drei Regionen wurden in den letzten Jahren in die KPCh aufgenommen. ${ }^{89}$

Zwischen dem 23. 6. und 3. 7. 1973 fanden Gewerkschaftskongresse in den Provinzen Guangdong, Hebei, Gansu, Shandong, Heilongiiang, Jiangsu, Shaanxi sowie dem Autonomen Gebiet Innere Mongolei statt. Frauen machten etwa 30 Prozent der Delegierten aus. Uber 220000 herausragende Arbeiter in den sieben Provinzen und dem einen Autonomen Gebiet wurden in den letzten Jahren in die KPCh aufgenommen.90

Die Wahl für die Komitees der Gewerkschaftsräte auf all diesen Kongressen sei, so wurde gesagt, in Einklang mit den fünf Anforderungen Mao's an die Fortsetzer der Revolution (1. Sie müssen wahre Marxisten-Leninisten sein. 2. Sie müssen Revolutionäre sein, die von ganzem

\footnotetext{
88 s. ebenda.

89 s. NCNA, 29.6.73.

90 s. NCNA, 6. 7.73.
} 
Herzen der Mehrheit des Volkes in China und der ganzen Welt dienen. 3. Sie müssen proletarische Staatsmänner sein, die fähig sind, sich mit der überwiegenden Mehrheit zusammenzuschließen und zusammenzuarbeiten. 4. Sie müssen Modelle für die Anwendung des demokratischen Zentralismus der Partei sein. 5. Sie müssen bescheiden und umsichtig sein und sich vor Arroganz und Ungestüm hüten; sie müssen erfüllt sein vom Geist der Selbstkritik und den Mut haben, Fehler und Unzulänglichkeiten in ihrer Arbeit zu verbessern) und dem Prinzip der Dreierverbindung von Älteren, Leuten mittleren Alters und Jüngeren erfolgt. Unter den Vorsitzenden der Provinzgewerkschaften befand sich eine Frau, und zwar in der Provinz Heilongjiang.

Nach dem Sieg über die ,,Viererbande“ im 11. Linienkampf der KPCh im Oktober 1976, einen Monat nach dem Tod Mao Zedong's, wurde eine erneute Sichtung der Reihen der Gewerkschaft und eine teilweise Reorganisierung erforderlich. Es wurde jedoch betont, daß trotz aller Abweichungen die revolutionäre Linie Mao Zedong's in der chinesischen Arbeiterbewegung vorherrschte und daß die Errungenschaften in der Gewerkschaftsarbeit größer gewesen seien als die Schwächen. Ferner sei die überwältigende Mehrheit der Gewerkschaftskader gut oder ziemlich gut. ${ }^{91} \mathrm{~W}$ as sind nun im einzelnen die Vorwürfe gegen die so bezeichnete ,,ultrarechte“ Fraktion in der Partei, die ,,Viererbande“, die unter Verwendung eines revolutionären Anstrichs an die Macht gelangen und in China eine ,Entwicklung“" durchsetzen wollte, die das Land auf den verschiedensten Gebieten um weitere Jahrzehnte zurückgeworfen hätte? Sie stigmatisierte die Gewerkschaften als ,, Gewerkschaften des ganzen Volkes", ,,Produktionsgewerkschaften“ und ,, Wohlfahrtsgewerkschaften“ und hetzte, man müsse ,,die alten Gewerkschaften zerschlagen. "92 Sie verfolgte Modellarbeiter und verdiente Arbeiter, die sie als ,,Leute mit angestammten Interessen“, ,,Konservative“ und als ,,die soziale Basis für die kapitalistischen Weggänger"93 verleumdete, sowie Gewerkschaftskader und Aktivisten. Sie wollte die Organisierung des sozialistischen Arbeitswettbewerbs und die Erwähnung von ,,Helden der Arbeit" verbieten. ${ }^{94}$ Die ,,Viererbande“ sei sogar dagegen gewesen, daß die Gewerkschaft marxistisch-leninistische Studien von Wissenschaft und Technik organisiere, und habe sich deren Rolle widersetzt, die Revolution anzupacken und die Produktion zu fördern. ${ }^{95}$ Letztendlich versuchte die ,,Viererbande“, die Gewerkschaften mit ihren eigenen Fraktionsorganisationen zu ersetzen und an die Stelle der Partei treten zu lassen, wodurch sie die Gewerkschaften über Partei und Regierung setzte, um sie zur Usurpierung der Führung von Partei und Staat zu mißbrauchen. ${ }^{\mathbf{9 6}}$ Ein Artikel in der ,,Volkszeitung“ beschäftigte sich speziell mit Wang Hongwen, dem ehemaligen Arbeiter. Er wurde kritisiert, die Führung der Partei über die Gewerkschaften negiert zu haben, indem er das Aushängeschild ,Führung durch die Arbeiterklasse“ benutzte. Er habe unter der Parole ,,den Klassenkampf als Hauptkettenglied anpacken“ (womit der Kampf der Bourgeoisie gegen das Proletariat gemeint sei) den Gewerkschaften verboten, der Sache der Partei und den Interessen der Arbeiter zu dienen. ${ }^{97}$

Der 9. Nationale Gewerkschaftskongreß wird im Oktober 1978 in Peking stattfinden. Dies wurde von der 7. (erweiterten) Tagung des 8. Exekutivkomitees des ACGB beschlossen, das vom 22.-24. 4. 1978 zum ersten Mal seit zwölf Jahren wieder tagte. Es nahmen fast 200 Leute

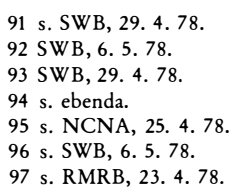


teil einschließlich der Vizevorsitzenden Ma Chungu, Zhu Xuefan, Li Jiebo, Gang Yonghe und Gu Dazhun. Ma sagte, wegen der Sabotage Lin Biao's und der ,,Viererbande“ sei solch ein Treffen nicht früher möglich gewesen. Der Gewerkschaftskongreß wird die Verfassung der chinesischen Gewerkschaft von 1957 revidieren und neue Führungsorgane des ACGB wählen. 98

Im März 1978 hatte der ACGB bereits etwa 100 vorbildliche Arbeiter und Gewerkschaftskader zu einer Aussprache zusammengerufen. Ma Chungu verglich die kommende Zeit mit dem legendären Langen Marsch der KPCh.99

In einem Rundschreiben des ACGB zur Einberufung des 9. Nationalen Gewerkschaftskongresses wurde die Gewerkschaft als unverzichtbare Organisation während der Ubergangsperiode vom Kapitalismus zum Sozialismus sowie als eine Säule der Diktatur des Proletariats, eine Brücke zwischen Partei und Massen und eine Schule des Kommunismus bezeichnet. Die Parteikomitees sollten die Führung über die Gewerkschaften noch verstärken. Mit dem Festhalten am Klassenkampf - der Kampf jetzt gegen den Einfluß der ,,Viererbande“müßten die Gewerkschaften geleitet werden. Als Hauptaufgaben der Gewerkschaftsarbeit wurden genannt: 1) die Propagierung der Linie und Hauptaufgabe für die neue Periode, wobei die Gewerkschaftsaktivisten eine große Rolle spielen;2) die Ermutigung der Arbeiter zur aktiven Kritik an der konterrevolutionären revisionistischen Linie der ,Viererbande“ (diese habe die Gruppe zur Vorbereitung der Einberufung des 9. Nationalen Gewerkschaftskongresses für ihre Ziele mißbraucht und einige Gefolgsleute zur Usurpierung der Gewerkschaftsmacht in mehreren Orten und Einheiten angestachelt) sowie zur Kritik an jedweden kapitalistischen Tendenzen; 3) Organisierung der Arbeiter zum Studium von Politik, Kultur, Wissenschaft und Technik sowie Leitung; Studienklassen wie auch FreizeitSchulen und ,,21. Juli““-Universitäten ${ }^{100}$ müßten gut organisiert werden; 4) Organisierung des sozialistischen Arbeitswettbewerbs, Herbeiführung eines neuen Aufschwungs in der sozialistischen Haltung ,,mit den Fortschrittlichen wetteifern, von ihnen lernen und sie einholen sowie den Zurückgebliebenen helfen", die Praktizierung von Sparsamkeit und zumindest die Erfüllung der staatlichen Pläne; 5) Hilfe für die Verwaltungsorgane bei der Leitung von kollektiven Wohlfahrtsprogrammen;6) Konsolidierung und Ausbau der Gewerkschaften, Ausrichtungsarbeit in den Betrieben, Heranziehung von Arbeitern an die Leitung der Betriebe. ${ }^{101}$

In der Maiausgabe 1978 des theoretischen Organs der KPCh ,,Rote Fahne“ (hongqi) ${ }^{\mathbf{1 0 2}}$ äusserte sich der Vizevorsitzende des ACGB Ma Chungu detailliert zur Frage der Gewerkschaften in dem Artikel ,,Gründlich die der Gewerkschaft gebührende Funktion entfalten“, wobei er auch auf das im Westen im Zusammenhang mit dem Sturz der ,Viererbande“ stark diskutierte Verhältnis von Produktion und Revolution einging. Gewerkschaften, so hieß es, seien auch in der Úbergangsperiode noch nötig, da das Proletariat zwar zur herrschenden Klasse geworden sei, der Klassenkampf aber noch weitergehe. Die Gewerkschaften müßten zur Schaffung solcher Bedingungen beitragen, die es möglich machten, daß die Bourgeoisie nicht mehr existieren und sich auch nicht mehr reproduzieren könne. Gerechte Forderungen und demokratische Rechte der Arbeiter sollten geschützt werden, weil es vorgekommen sei, daß eine bürokratische, kapitalistische Wirtschaftsverwaltung ihre Interessen verletzt habe. Wenn Arbeiter aus Prinzipientreue dagegen anzukämpfen wagten, hätten sie manchmal

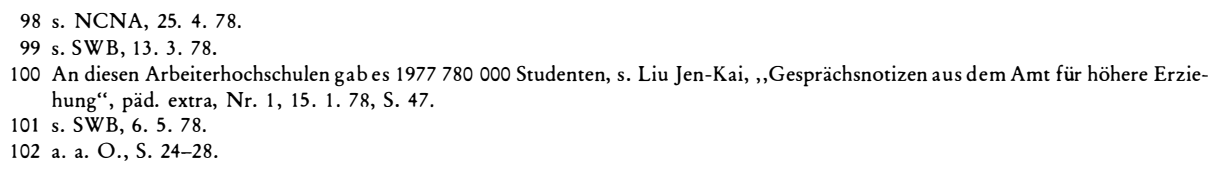


noch Vergeltungsschläge einer falschen Führung hinzunehmen. Zur Entfaltung der Funktion der Gewerkschaften sei es unbedingt erforderlich, daß diese an den ,,drei großen revolutionären Bewegungen“, Klassenkampf, Produktionskampf und wissenschaftliches Experiment, festhielten. Zum Problem der ,,Viererbande“ hieß es, daß sie unter dem Vorwand des Klassenkampfes das Proletariat angegriffen und sowohl die Produktion als auch die Revolution und die Gewerkschaftsarbeit sabotiert habe. Mit ihrer Festlegung, daß es Aufgabe der Gewerkschaft sei, mit denen in der Partei, die den kapitalistischen Weg gingen, zu kämpfen, habe sie in ihrem Artikel in der ,,Volkszeitung“ vom 21. Mai 1976 die „Beziehungen zwischen uns und unseren Feinden“" auf den Kopf gestellt. ,,Wenn man nicht den Klassenkampf als Hauptkettenglied nimmt, dann wird man die Orientierung verlieren, und die einzelnen Arbeiten werden bestimmt nicht gut erledigt werden können. Wenn man nur am Klassenkampf festhält und nicht die anderen Arbeiten anpackt, nicht die Reihen der Arbeiter und Angestellten aufbaut, nicht gut die Produktion betreibt und sich nicht um das Leben der Massen kümmert, bekommt man den Klassenkampf auch nicht gut in den Griff, und das Entfalten der der Gewerkschaft gebührenden Funktion wird zu leerem Geschwätz.“ „,Hält man nicht am Produktionskampf fest, entwickelt man nicht mit hohem Tempo die sozialistische Wirtschaft, hat die Diktatur des Proletariats keine starke materielle Basis, wie kann man dann noch von der Konsolidierung der Diktatur des Proletariats reden? Die Viererbande stellte Revolution und Produktion in einen Gegensatz; wenn die Gewerkschaft die Arbeiter und Angestellten zur guten Erledigung der Produktion mobilisierte, verleumdete sie sie als ,Produktionsgewerkschaft'. “ Man müsse, so Ma Chungu, sowohl gegen den Bürokratismus kämpfen, der sich nicht um die Sorgen der Massen kümmere, als auch gegen den OÓkonomismus, der nicht die Staatsinteressen berücksichtige, sondern einseitig die Wohlfahrt betone. Grundlegendste regelmäßige Aufgabe der Gewerkschaft sei es, eine in hohem Maß revolutionierte und mit der Technik vertraute industrielle Armee aufzubauen.

Im Hinblick auf die vielschichtige Entwicklung der Gewerkschaften seit Gründung der VR China müssen der 9. Nationale Gewerkschaftskongreß im Oktober 1978 und die künftige Gewerkschaftsarbeit mit Interesse verfolgt werden. Sicher ist, daß sich an der Führung der Gewerkschaft durch die Partei nichts ändern wird und daß die Gewerkschaften einen wichtigen Beitrag zur Verwirklichung der von Zhou Enlai in Übereinstimmung mit den Prinzipien Mao Zedong's für den Aufbau des Sozialismus propagierten ,,vier Modernisierungen“, die Hauptaufgabe für die chinesische Gesellschaft bis zum Jahr 2000, zu leisten haben.

\section{Gespräche mit Gewerkschaftsführern aus Kanton ${ }^{103}$}

Während der Kulturrevolution waren die Gewerkschaftsorganisationen auf der oberen Ebene zerstört, aber an der Basis existierten sie noch. Einige nationale Gewerkschaften waren der Leitung einer regionalen Gewerkschaft unterstellt. Der Arbeiterdelegierten-Kongreß, der nach dem 9. Parteitag der KPCh an die Stelle der Gewerkschaft trat, hatte unklare Aufgaben und Ziele. Es war eine Organisationsform für die Úbergangsperiode, sie existierte nur auf Provinz-, Stadt- und Bezirksebene.

Der Vorsitzende der Gewerkschaft ist auch Mitglied des Parteikomitees. 85 bis 90 Prozent der Arbeiter sind gewerkschaftlich organisiert. Der Mitgliedsbeitrag ist nach dem Lohn ge-

103 Als Teilnehmer einer Reisegruppe der Gesellschaft für deutsch-chinesische Freundschaft (GDCF) erhielt ich am 7.8.78 in Kanton die Gelegenheit zu einem dreistündigen Gespräch mit vier kantonesischen Gewerkschaftsführern, u. a. mit dem Leiter der Abteilung Produktion der Zentralgewerkschaft Kanton. An dieser Stelle werden die wichtigsten Punkte kurz wiedergegeben.

Dr. Helmut Martin und Peter Schier, Institut für Asienkunde Hamburg, sei hier für die Bereitstellung einigen Materials gedankt. 
staffelt: Wer unter 50 yuan verdient, zahlt einen mao; bei über 50 yuan - zwei mao; bei über 100 yuan - drei mao. Auch Lehrlinge können nach einiger Zeit Mitglied werden. Sie zahlen fünf fen. Ist ein Mitglied über ein halbes Jahr krank, bekommt es den vollen Lohn weiter, ein Nichtmitglied dagegen nur 50 Prozent. Angehörige von Gewerkschaftsmitgliedern erhalten im Krankheitsfall 50 Prozent Unterstützung. Beim Tod eines Mitglieds werden zwölf Monatslöhne an die Familie gezahlt.

In der Gewerkschaft gibt es eine Abteilung Frauen, dafür existiert der Frauenverband auf Fabrikebene nicht.

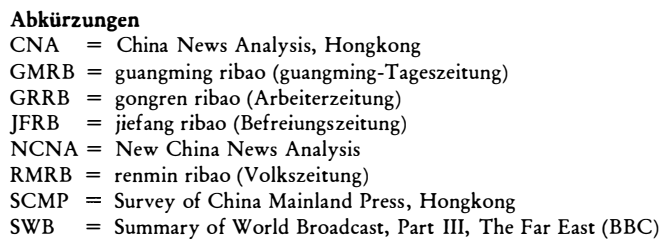




\section{"Government Policies Toward Unions and Dependent Development."}

B) A. BolichH

The study of government policies toward unions in Venezuela, Colombia and Peru is preceded by an investigation of the economic parameters of such policies. For each country, the specifics of its dependent economic structure are outlined. Particular attention is paid to changes with respect to capital intensity of investments, productivity, degrees of competition, income, and income distribution within economic sectors and the relationship between these sectors. Thus, some understanding of the forces shaping the labor markets can be achieved.

The results can be summarized as follows: In Venezuela, the abundance of capital and the extremely oligopolistic structure of the foreign oriented, highly capitalized sector has resulted in a rigid separation of economic sectors and labor markets, negative income and growth effects for the undercapitalized sector, and in the fact, that in the highly capitalized sector wages do not affect profits. In Colombia and Peru, on the other hand, the economic margins for union activities are much smaller. However, the separation of the economic sectors seems to be less rigid than in Venezuela, and the growth of the highly capitalized sector generally has positive growth and income effects for the rest of the economy.

During the various stages of development, the labor and union policies of governments have been influeneed by considerations of preventive conflict solution, the role of unions as political resources, and the attempts of governments to adjust to or solve the problems of dependent development. It can be shown that the specific mixture of motives and the particular limitations of government policies toward modern labor and unions are largely determined by the economic parameters of the different stages of dependent development.

\section{"Labor Law and Labor Relations in Chile 1900-1970. . ."}

By HeleEn F.-P. IETSWAaR'T

The article first describes how in Chile developed, during the first two decades of this century, a system of labor relations, and how then the economically dominant classes tried to change this system through legislation, thus requiring substantial changes in the behavior patterns of the social groups concerned. The analysis then focusses on how the interaction between those legal prescriptions and the concrete social and political forces as they developed has over time led to the particular mixture of legal, illegal and a-legal behavior which characterizes labor relations in Chile in the late 1960's, and emphasizes the relative importance of the legal system for an adequate understanding of the development of those labor relations.

\section{Trade Unions in the People's Republic of China By LiU JeN-KaI}

In the People's Republic of China, a country which is applying Marxist-Leninist principles on politics and economics, trade unions are considered as a transmission belt between the Communist Party and the masses and a school where the workers learn management and learn communism. There have been several conflicts due to "economism" and "syndicalism". It is the duty of the trade unions to educate the workers to understand that there 
should be an identity between the long-term interests of the state and the interests of the workers who are the leading class in the country. It was not always easy for union cadres, many of them Party members, to carry out Party policies on the one hand and to stand up for the immediate interests of the workers on the other hand. Some leading Party members like Liu Shaoqi and the "gang of four" tried to falsify the function of the unions in order to use them for their own purpose of usurping Party and state power. The basic principles for effective trade union work is to adhere to the leadership of the Party and to rely firmly on the masses. The basic constant task is to build an industrial army which is highly revolutionized and well versed in technical science. So far there have been two All-China Trade Unions Congresses of the PRC, the 7th (1953) and the 8th (1957). The 9th will be convened in October, 1978. The main task of the workers will be the implementation of the "four modernizations" in order to build a great and powerful socialist country with modernized agriculture, industry, national defence and science and technology before the end of the century.

\section{Thirty Years of Industrial Relations Legislation in Ghana: 1941-1971 \\ By Akilagpa SaWyer}

This review of legislation governing the organisation of trades unions in Ghana in the period 1941-71 deals with the ways in which the varying and conflicting positions taken by government administrative and political agencies, the unions themselves and external bodies like the British Tuc, the American AFL-CIO and the ICFTY influenced the form and substance of such legislation.

In the period before political independence the colonial administration sought to keep the labour movement out of political activity, whilst the CPP and the dominant elements in the movement worked for its active involvement. In pursuance of this latter object the CCP backed a process of amalgations to ensure the centralisation of control and the strengthening of the workers movement. This process came up against not only centrifugal tendencies within the movement itself, but also the activities of colonial administration, political opposition to the CPP, and the external bodies mentioned above. To counteract these forces the CPP government one year after independance enacted legislation concentrating power and funds in a centralised Trades Union Congress (TYC), which later became an "auxilliary wing" of the CPP. The role of the TYC was to bring the workers behind the government policy and ensure harmony on the industrial scene. After the overthrow of the CPP in 1966 the succeeding NLC and PP regimes sought to break the hold of CPP elements over the labour movement and weaken the opposition of the workers to their policies, by removing the legislative basis of TUC control and depoliticising the labour movement generally.

An interesting feature of this review is the light it sheds on the circumstances under which particular programmes of legislation are effective, in the sense of achieving their main objectives. For instance, the colonial administration's attempt to legislate the labour movement out of the political arena was a failure, while the CPP government was later succesful in legislating centralisation of the labour movement. The failure of the colonial attempt was largely due to the fact that it ran counter to the general anti-colonial activism of the time, and was not backed by adequate political action. The CPP success, on the other hand, is explained by the circumstance that the legislation was backed by such institutional arrangements as the denial of legitimacy and funds to non-Tuc unions, and formed part of the general move towards making Ghana a one-party state. 